\title{
Observations of urban boundary layer structure during a strong urban heat island event
}

Article

Accepted Version

Author copy - equations not clear, contact author if in doubt

Barlow, J. F., Halios, C. H., Lane, S. E. and Wood, C. R. (2015) Observations of urban boundary layer structure during a strong urban heat island event. Environmental Fluid Mechanics, 15 (2). pp. 1567-7419. ISSN 1567-7419 doi: https://doi.org/10.1007/s10652-014-9335-6 Available at https://centaur.reading.ac.uk/38570/

It is advisable to refer to the publisher's version if you intend to cite from the work. See Guidance on citing.

Published version at: http://dx.doi.org/10.1007/s10652-014-9335-6

To link to this article DOI: http://dx.doi.org/10.1007/s10652-014-9335-6

Publisher: Springer

All outputs in CentAUR are protected by Intellectual Property Rights law, including copyright law. Copyright and IPR is retained by the creators or other copyright holders. Terms and conditions for use of this material are defined in the End User Agreement.

www.reading.ac.uk/centaur 
Central Archive at the University of Reading

Reading's research outputs online 


\title{
Observations of urban boundary layer structure during a strong urban heat island event
}

\author{
J. F. Barlow ${ }^{1}$, C. H. Halios ${ }^{1}$, S. E. Lane ${ }^{2}$ and C. R. Wood ${ }^{1,3}$ \\ (1) Department of Meteorology, University of Reading, PO Box 243, Reading, RG6 6BB, UK \\ (2) Department of Meteorology, Met Office Research Unit (Cardington), Cardington Airfield, Shortstown, \\ Bedfordshire, MK42 0SY, UK \\ (3) Finnish Meteorological Institute, Erik Palménin aukio 1, Helsinki , 00101, Finland
}

J. F. Barlow Email: j.f.barlow@reading.ac.uk

\begin{abstract}
It has long been known that the urban surface energy balance is different to that of a rural surface, and that heating of the urban surface after sunset gives rise to the Urban Heat Island (UHI). Less well known is how flow and turbulence structure above the urban surface are changed during different phases of the urban boundary layer (UBL). This paper presents new observations above both an urban and rural surface and investigates how much UBL structure deviates from classical behaviour. A 5-day, low wind, cloudless, high pressure period over London, UK, was chosen for analysis, during which there was a strong UHI. Boundary layer evolution for both sites was determined by the diurnal cycle in sensible heat flux, with an extended decay period of approximately $4 \mathrm{~h}$ for the convective UBL. This is referred to as the "Urban Convective Island" as the surrounding rural area was already stable at this time. Mixing height magnitude depended on the combination of regional temperature profiles and surface temperature. Given the daytime UHI intensity of $1.5^{\circ} \mathrm{C}$, combined with multiple inversions in the temperature profile, urban and rural mixing heights underwent opposite trends over the period, resulting in a factor of three height difference by the fifth day. Nocturnal jets undergoing inertial oscillations were observed aloft in the urban wind profile as soon as the rural boundary layer became stable: clear jet maxima over the urban surface only emerged once the UBL had become stable. This was due to mixing during the Urban Convective Island reducing shear. Analysis of turbulent moments (variance, skewness and kurtosis) showed "upside-down" boundary layer characteristics on some mornings during initial rapid growth of the convective UBL. During the "Urban Convective Island" phase, turbulence structure still resembled a classical convective boundary layer but with some influence from shear aloft, depending on jet strength. These results demonstrate that appropriate choice of Doppler lidar scan patterns can give detailed profiles of UBL flow. Insights drawn from the observations have implications for accuracy of boundary conditions when simulating urban flow and dispersion, as the UBL is clearly the result of processes driven not only by local surface conditions but also regional atmospheric structure.
\end{abstract}

Keywords Urban boundary layer Doppler lidar Urban heat island Turbulence profiles 


\section{Introduction}

It has long been known that the urban surface energy balance is different to that of a rural surface, and that prolonged heating of urban surfaces after sunset gives rise to the Urban Heat Island (UHI). Daytime sensible heat fluxes are generally larger over the drier urban surface, leading to deeper urban boundary layers (UBL). What is less well known is how fundamentally the structure of the flow and turbulence above the urban surface is changed during different stability phases of the UBL, in particular during the peak of the UHI after sunset.

There have been few examples of simultaneous surface observations and boundary layer profiles in an attempt to relate UBL structure to UHI processes [ $\underline{1}, \underline{2}]$. For instance, Pal et al. [ $\underline{3}]$ observed a deeper nocturnal boundary layer over the urban area in Paris compared to suburban and rural sites, but with large spatial variability in depth which correlated with the UHI pattern. During the day, a deeper boundary layer and thicker entrainment zone were observed over the urban area, and UBL decay rates were slower over the urban area and attributed to increased thermal inertia in the urban surface. Nocturnal jets have been observed over urban areas [ $4 \underline{]}$ and there is evidence of a change in their structure due to advection over the urban surface [ $\underline{5}$ ]: the jet maximum tends to be higher up over the urban area. Turbulence structure beneath nocturnal jets is the result of a complex interaction with the urban surface and simulation of dispersion in such flows remains difficult [ㅁ] .

A lack of measurements above the urban surface layer has previously limited investigation of UBL mixed/residual layer characteristics [7]. Remote sensing techniques such as sodar and lidar have led to greater understanding of urban flows (see $[\underline{8}]$ for review). There has been much work done on deriving mixing heights $[\underline{9}, \underline{10}]$, as tethered balloons or radiosondes tend not to be used in dense urban areas due to practical restrictions. Dopplerized instruments give direct observations of wind-speed and turbulence which can lead to greater modelling accuracy when assimilated e.g. [11]. Doppler lidars in particular have great potential to observe the wind and turbulence profiles throughout the UBL which are needed within dispersion models $[\underline{12}, \underline{13}]$. Based on a small amount of data, Doppler lidar profiles of vertical velocity variance have been shown to resemble the classical structure for both convective and neutral boundary layers $[\underline{8}]$. Further work is thus required to fully exploit their potential in urban areas, especially in combination with other observations.

The lack of simultaneous observations of UBL structure and fluxes over a longer period motivated the Advanced Climate Technology Urban Atmospheric Laboratory (ACTUAL) project in London, UK. This project established instrumentation platforms at two heights in the urban area with Doppler lidar observations of UBL structure for the period spring 2010 to winter 2012, in parallel to existing flux and lidar measurements at a rural site. This paper presents ACTUAL observations taken during an unusually cloudless, low wind, high pressure period when differences in surface energy balance for the urban and rural surfaces were assumed to be large. The observations are used to establish the differences in rural and urban boundary layer evolution. Night-time lidar observations of wind profiles over the urban area are then used to assess the behaviour of the nocturnal jet, and to test whether prolonged mixing after sunset has an impact on jet evolution. Finally, daytime wind and turbulence profiles in different phases of the urban convective boundary layer are compared to classical results.

\section{Methodology}

\subsection{Experimental set-up}

Figure 1 shows the ACTUAL project measurement sites in London: the BT Tower (latitude $51 \circ 31^{\prime} 17.6^{\prime \prime} \mathrm{N}$ longitude 0॰08'20.36”W) and the Westminster City Council (WCC) roof-top site (latitude 51॰31'16.31'N longitude $\left.0 \circ 09^{\prime} 38.33^{\prime \prime} \mathrm{W}\right)$. The BT Tower is the tallest building within several kilometres, with good exposure to winds in all directions. Within $10 \mathrm{~km}$ of the BT Tower the land surface cover is a mixture of residential and commercial with a mean building height of $8.8 \mathrm{~m}$, roughness length within central London is estimated to be $0.87 \pm 0.48 \mathrm{~m}$, and the displacement height $4.3 \pm 1.9 \mathrm{~m}[\underline{14}]$. There are two large parks nearby: Regent's Park $(1.66 \mathrm{~km} 2)$ approximately 
$0.64 \mathrm{~km}$ north-west of the Tower; Hyde Park $(2.53 \mathrm{~km} 2)$ approximately $1.7 \mathrm{~km}$ to the south-west. It should be noted that due to the very local nature of the WCC measurement (its source area lies mostly within $200 \mathrm{~m}$ of the rooftop on which it is measured as determined from wind-tunnel experiments, A. Brocklehurst, personal communication), measurements are representative of the urban surface, whereas the source area for the BT is much larger $(2-3 \mathrm{~km}$ in convective conditions, $10-20 \mathrm{~km}$ in neutral [ $\underline{15}]$ ), and represents a mixture of urban and vegetative surfaces.

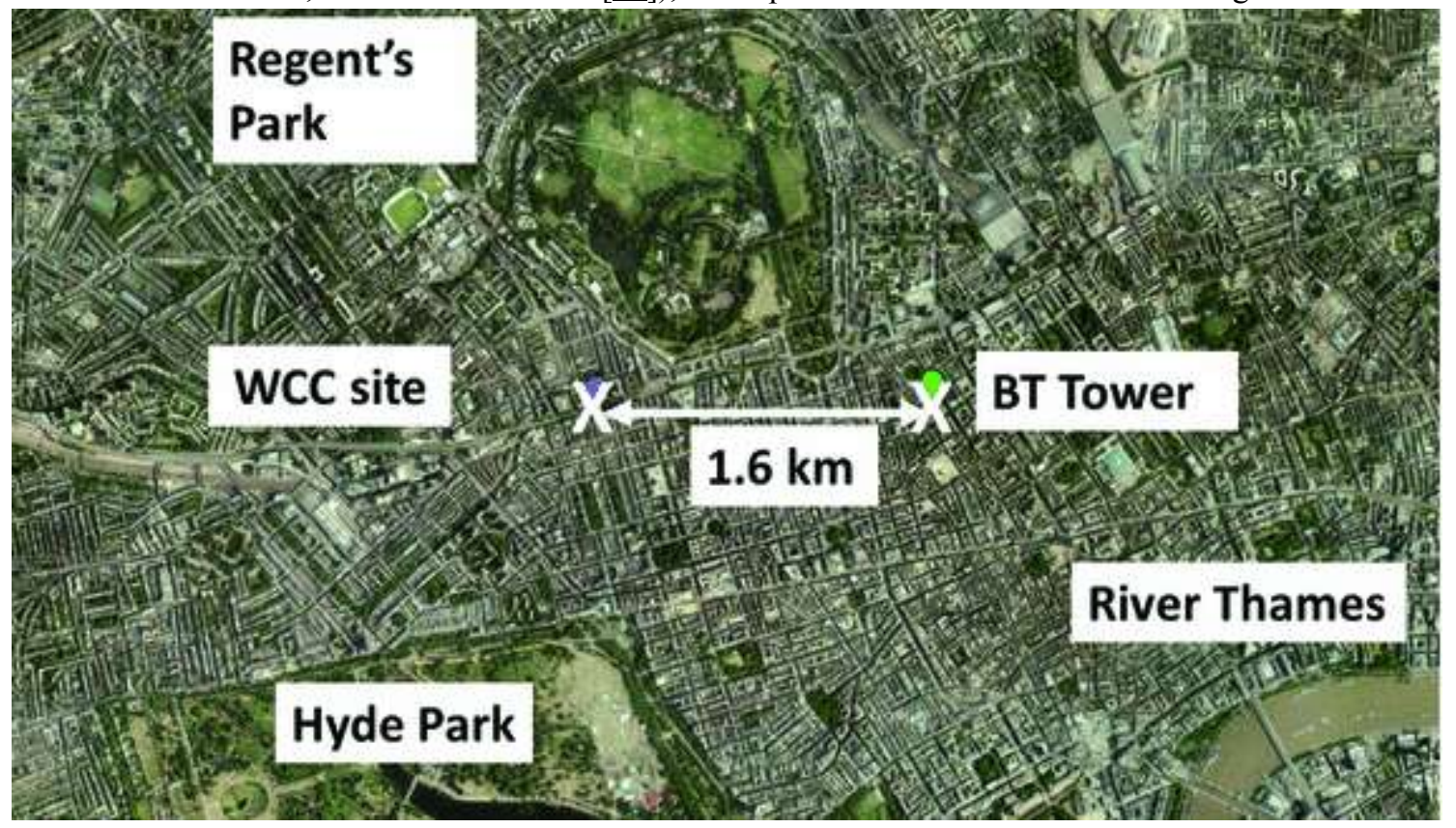

Fig. 1 Map of central London showing BT tower and WCC measurement sites

A heterodyne Doppler lidar (Halo-Photonics "Streamline") with scanning capability was installed on the roof-top of the WCC site to measure the vertical structure of the UBL. The instrument operated at $1.5 \mu$ mwavelength; the pulse repetition frequency was $20 \mathrm{kHz}$, with integrated signals being outputted every $3.6 \mathrm{~s}$; the sampling frequency was $30 \mathrm{MHz}$, and the return signal was resolved into $30 \mathrm{~m}$ long range gates. Within the first three gates of the lidar $(90 \mathrm{~m})$, returns were of insufficient quality due to transmitter and receiver not being co-located; therefore results are presented from the fourth gate upwards, mid-gate height being $124 \mathrm{~m}$ a.g.l. once height of the WCC building at the location of the lidar is taken into account.

Two modes of operation were used: (a) continuous stare mode (pointing vertically) and (b) Doppler Beam Swinging (DBS) mode for measuring the vertical wind profile. The DBS mode sampled in three orthogonal directions: vertically, tilted 15。 off-zenith to east and to north. The DBS scan cycle lasted approximately $21 \mathrm{~s}$ and the time interval between the start of scans in DBS mode was $120 \mathrm{~s}$, and the lidar was in vertical stare mode in the intervening $99 \mathrm{~s}$. Resulting hourly averaged wind-speeds had previously been compared with the BT Tower sonic anemometer measurements over a 3,993 h-long period and were found to have a near 1:1 (0.99) correlation [ $\underline{16}]$.

Alongside the lidar, two identical instrumentation platforms were used for the turbulent flux measurements; these platforms were equipped with identical instrumentation: sonic anemometer (Gill Instruments R3-50), hygrometer (Licor Li-7500), net radiometer (Kipp and Zonen CNR4) and weather station (Vaisala WXT520). The head of each sonic anemometer was placed respectively at the WCC site at a height of $3 \mathrm{~m}$ above the roof-top of $15 \mathrm{~m}$ height, and at $191 \mathrm{~m}$ on the BT Tower. At the BT Tower, the sonic anemometer was clamped to a pole on the top of an open lattice scaffolding tower of $12.3 \mathrm{~m}$ height on top of the main structure, meaning that the sensor head was $0.76 \mathrm{~m}$ higher than the lattice, at which level the rest of the instrumentation was mounted, the net radiometer being on a $0.5 \mathrm{~m}$ long horizontal boom. A full description of the lattice is given in [1]], where it was deduced from wind tunnel tests that there is only slight flow distortion at the height of the sensor due to the lattice. At the WCC site, the anemometer is mounted at the location first described by Barlow et al. [18] as used in the DAPPLE project (referred to as the "LIB" reference in that paper, here labelled "WCC"). Buildings in the vicinity are densely packed (plan area density $\lambda_{p} \sim 0.4$ ), a mixture of residential and commercial buildings, and mean building height within a 250 m radius is $21 \mathrm{~m}$. 
The rural boundary layer was monitored using observations made at the Chilbolton Facility for Atmospheric and Radio Research (CFARR) in southern England (51॰09'N,01॰26'W). A Doppler lidar (Halo Photonics), an earlier model of the one deployed in central London, was operated in continuous vertical stare mode. This lidar had a range-gate spacing of $36 \mathrm{~m}$ and a nominal time resolution of $40 \mathrm{~s}$. Also, measurements were available from a sonic anemometer (Metek USA-1) and an open-path gas analyser (LICOR Li-7500) mounted at a height of $5.3 \mathrm{~m}$ a.g.1. A pyranometer (Kipp and Zonen CNR1) was mounted at approximately $2 \mathrm{~m}$ a.g.1. All sensors were located $200 \mathrm{~m}$ away from the lidar over grass surface. Surrounding land cover was predominantly agricultural fields and terrain was reasonably flat.

Data from radiosonde launches at Larkhill $\left(51 \circ 12^{\prime} 0^{\prime \prime} \mathrm{N}, 1 \circ 48^{\prime} 0^{\prime \prime} \mathrm{W}, 25 \mathrm{~km}\right.$ West-South-West of Chilbolton and $125 \mathrm{~km}$ West-South-West of London) and Herstmonceux (50॰53'60"N,0॰19'48"E, 78 km South-South-East of London and $6 \mathrm{~km}$ inland) were also available. These soundings were used to give boundary layer temperature profiles in the rural areas around London.

Surface weather station data taken from the UK Met Office observation network MIDAS were used. Screen temperature measured at $1.2 \mathrm{~m}$ was used at several sites to calculate the UHI intensity.

Note that all averaged data are time-stamped at the end of the period, i.e. 01:00 is data averaged from 00:01 to 01:00.

\subsection{Eddy covariance data processing}

The three wind components $(u, v, w)$ and sonic temperature $\left(T_{S}\right)$ from each sonic anemometer were archived at $20 \mathrm{~Hz}$. No corrections were applied to the data (such as path averaging, spectral loss or cosine response). Following earlier work at the BT site [ $\underline{14}] 30$ min periods were calculated to be long enough to capture most of the scales of turbulence contributing to fluxes across most periods; within this paper the averaging period is variously 30 or 60 min according to the analysis presented. Data were subject to quality control: data were removed if (i) the period of 30 min was not complete; (ii) the percentage of spikes exceeded $0.5 \%$; (iii) the spectra of $u, v, w$ or $T$ exhibited high-frequency noise; (iv) there was an extreme vertical rotation angle of greater than 2.5 standard deviations (SD) from the mean. Spikes were defined as short duration $(<0.1 \mathrm{~s})$ and large amplitude $(>5 \mathrm{SD})$ fluctuations in the time series, resulting from random electronic noise or sensor error caused by rain or other airborne matter (such as biological agents). To calculate fluxes, block averages were used to define fluctuations and double-rotation was used [ 19$]$ so that the mean vertical and span-wise wind speeds were defined as zero $\left(v^{-}=w^{-}=0\right)$ for each averaging period. Sensible heat fluxes were computed using the sonic temperature. Data from the slow response sensors (net radiometer, weather station) were logged at $1 \mathrm{~Hz}$.

\subsection{Doppler lidar data processing}

Due to the limited sampling rate of the lidar, a spectral correction was applied to each averaging period to account for underestimation of the vertical velocity variance, $\sigma 2 \mathrm{w}$ (e.g. see [20]). Due to the lidar's scan schedule, approximately 30 sub-periods each containing 25 measurements of $w$ were taken each hour. A Fourier Transform was applied to the $w$ time series for each sub-period to obtain a power spectrum. Then the hourly average of these spectra was calculated to give the spectral density between $f_{\min }=1 /(25 \Delta t)$ and Nyquist frequency $f_{N y}=1 /(2 \Delta t)$. Following the method of Hogan et al. [20], it was assumed that the last five points of the average spectral density estimates lie in the inertial sub-range, where

$S(f)=S 0 f-53$

and $S_{0}=\alpha \varepsilon-2 / 3$ ( $\alpha$ is taken to be 0.5$)$. Note that $4 S_{0}$ is the structure parameter. The observations should be corrected to allow for the actual variance being given by the observed variance plus an additional factor:

$\sigma 2 w=w^{\prime} 2+\epsilon$,

where $\epsilon$ is the unsampled variance at frequencies higher than the Nyquist frequency and is given by 
Note that the equation is expressed in terms of sampling interval $\Delta t$ for direct comparison with Eq. ( $\underline{3})$ in [20]. The coefficient in Eq. (ㄱ) above has been corrected to give a correction factor approximately four times larger than given in $[\underline{20}]$.

To evaluate the correction factor, Fig. $\underline{2}$ shows a scatterplot of $\sigma 2 \mathrm{w}$ for the lidar gate nearest in height to the BT Tower (i.e. gate 6 with mid-point $184 \mathrm{~m}$ ) against the BT Tower sonic anemometer data. Three data pairs are presented:

(1) Uncorrected lidar data $\left(f_{s \_l i d}=0.278 \mathrm{~Hz}\right)$ against sonic anemometer data $\left(f_{s_{-}}\right.$son $\left.=20 \mathrm{~Hz}\right)$

(2) Uncorrected lidar data $\left(f_{s \_l i d}=0.278 \mathrm{~Hz}\right)$ against degraded sonic anemometer data $\left(f_{s \_s o n}=0.278 \mathrm{~Hz}\right)$

(3) Corrected lidar data against sonic anemometer data $\left(f_{s \_s o n}=20 \mathrm{~Hz}\right)$

In Case 1, without correction it can be seen that the uncorrected lidar variance is linearly correlated with the sonic derived data, but with some scatter $\left(R_{2}=0.79\right)$ and underestimated compared to a $1: 1$ relationship $($ slope $=0.72)$. In Case 2, the sonic anemometer is degraded to the same sampling rate as the lidar, thus taking into account the difference due to different sampling rates (as done by Barlow et al. [ $\underline{8}]$ ). The slope increases to 0.79, which is logical as matching the sampling rates should improve the relationship to the best agreement possible, given difference in sampling volume (point measurement vs. spatial averaging over the lidar gate), and spatial separation of the instruments. Case 3 is a comparison of lidar data corrected using Eq. ( $\underline{3}$ ) with sonic data sampled at $20 \mathrm{~Hz}$. It can be seen that the slope is also increased to 0.80 which is statistically indistinguishable from Case 2. This gives confidence that the correction procedure for the lidar variance is reasonable. Although the average improvement is $8 \%$, on individual points the correction varies between less than $5 \%$ during daytime up to approximately $45 \%$ during the night when turbulence length-scales are small. This indicates that the correction is worth applying.

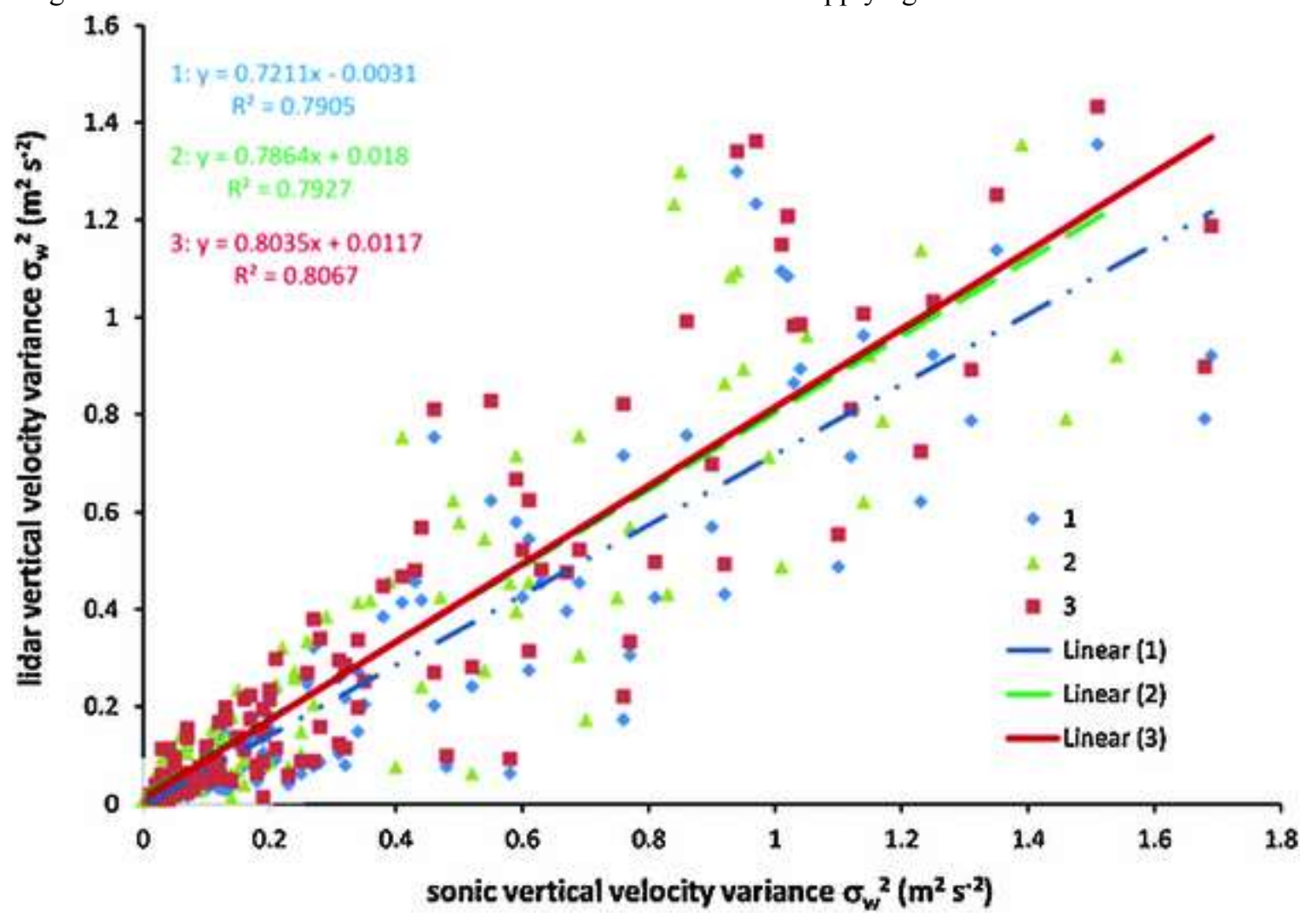

Fig. 2 Scatter plot of vertical velocity variance $\sigma 2 w$ measured using Doppler lidar against sonic anemometer at BT Tower. Case 1 uncorrected lidar against sonic anemometer data; Case 2 uncorrected lidar data against degraded sonic anemometer data (i.e. matched sampling rate); Case 3 corrected lidar data against sonic anemometer data 
After correcting the variance data, mixing heights $(\mathrm{MH})$ were derived from lidar turbulence measurements by simply considering a threshold value of $0.1 \mathrm{~m} 2 \mathrm{~s}-2$ [ $\underline{8}]$. In order to check the sensitivity of the calculated height to changes in the threshold, a $30 \%$ perturbation of the threshold value was applied. In particular, for each hour 21 values of the $\mathrm{MH}$ were estimated considering 21 threshold values ranging from $0.069 \mathrm{~m} 2 \mathrm{~S}-2 \mathrm{up}$ to $0.129 \mathrm{~m} 2 \mathrm{~S}-2$. Thus the final estimate of $\mathrm{MH}$ was taken to be the average and the uncertainty range is given by the minimum and maximum values. Differences between MHs obtained from the different threshold values averaged at $6 \%$ for the London site. It should be noted that the minimum observable $\mathrm{MH}$ that could be obtained with this method was $124 \mathrm{~m}$ a.g.l. (=mid-gate height) for London and $162 \mathrm{~m}$ a.g.l. for Chilbolton, and so during the night-time the actual $\mathrm{MH}$ was sometimes lower than the minimum observable height.

\section{Differences in rural and urban boundary layer evolution}

Simultaneous observations at the rural (Chilbolton) and urban (WCC, BT) sites are now compared to establish the difference in rural and UBL evolution.

\subsection{Synoptic conditions and temperature profiles}

During the chosen study period from 28 September to 2 October 2011, a high pressure system was centred over Europe, resulting in unusually cloudless skies, light southerly sector winds, and temperatures that were warmer than average over the UK. Figure $\underline{3}$ shows temperature profiles measured by radiosondes released at Larkhill and Herstmonceux. As the high pressure system was a fairly stationary feature, there is little difference spatially between the profiles at both sites despite $150 \mathrm{~km}$ separation, and temporally at the different times of observation each day above the surface layer, i.e. the lowest $400 \mathrm{~m}$. The most distinctive change occurred between 28 and 29 September when there was

a 3。 warming of the boundary layer.

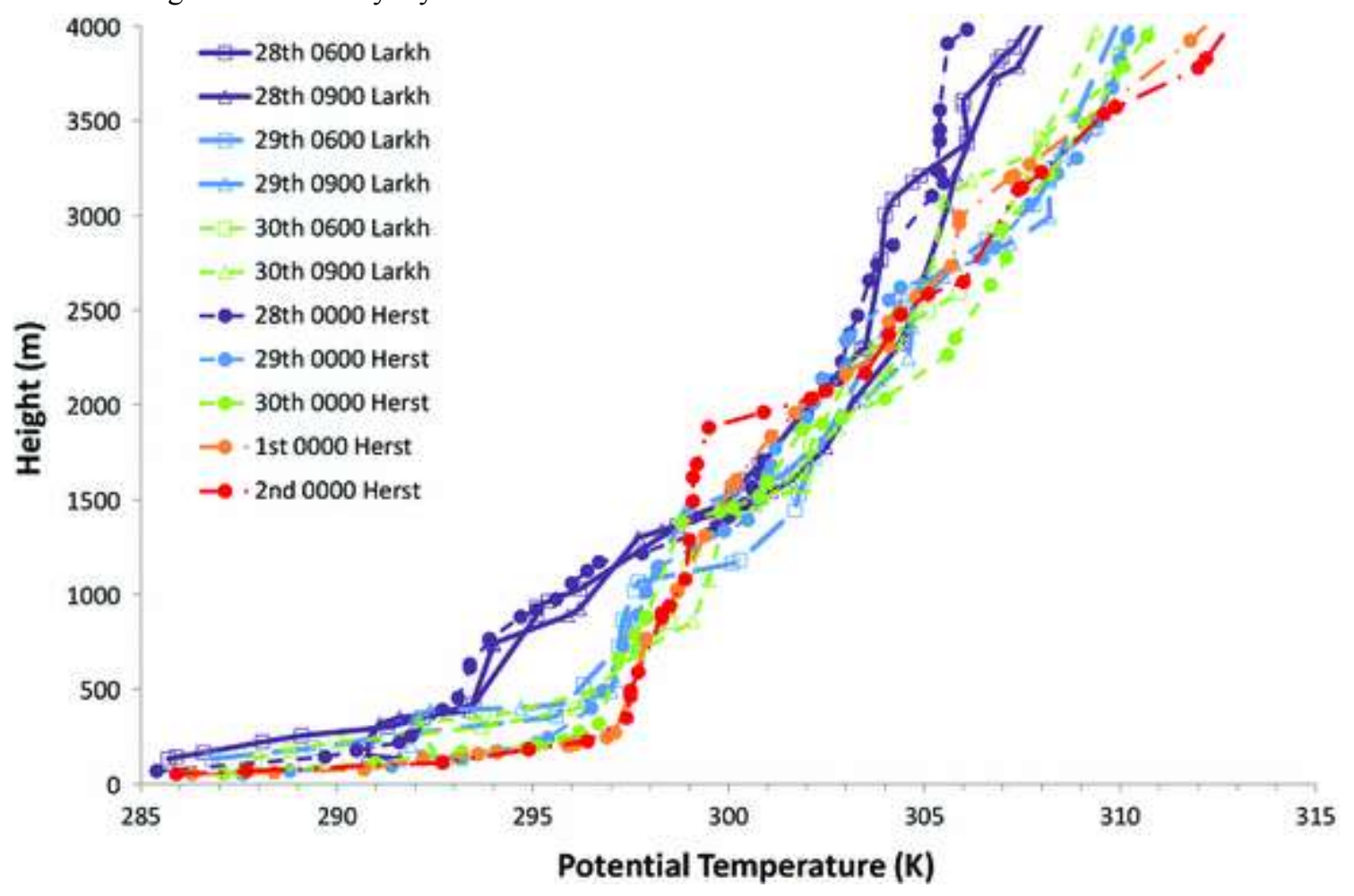

Fig. 3 Potential temperature profiles throughout study period (28 September-2 October) measured using radiosonde profiles from Larkhill (continuous line) and Herstmonceux (dashed line) 


\subsection{Urban heat island intensity}

UHI intensity was defined as the air temperature difference between urban and rural stations. Figure $\underline{4}$ shows the UHI calculated using six urban stations (Hampstead, Kenley Airfield, Kew, Heathrow Airport, Saint James Park, Northolt) and Chilbolton as the rural reference site. In most of the cases a clear diurnal cycle with high positive values during night-time and mostly small, positive values during the day can be observed, in accordance with other studies (e.g. [21]). Differences between the sites are due to local surface energy balance and possible advection [22] . It is interesting to notice that the amplitude of the oscillation is particularly pronounced for Kenley, which is located at the southern edge of the city. At Heathrow and St. James's Park UHI intensity is of a similar magnitude. At Kew and Northolt the UHI signal is highly variable, due to local land use characteristics. In general UHI intensity for all stations is stronger over the last 2 days, which may be associated with drying of the ground and vegetation, all sites having between 10 and $30 \%$ vegetated land-use fraction within $50 \mathrm{~m}$ of the site. On average, the urban surface is approximately $4 \circ \mathrm{C}_{\text {warmer }}$ than the rural site by night, and $1.5 \circ \mathrm{C}$ warmer by day.

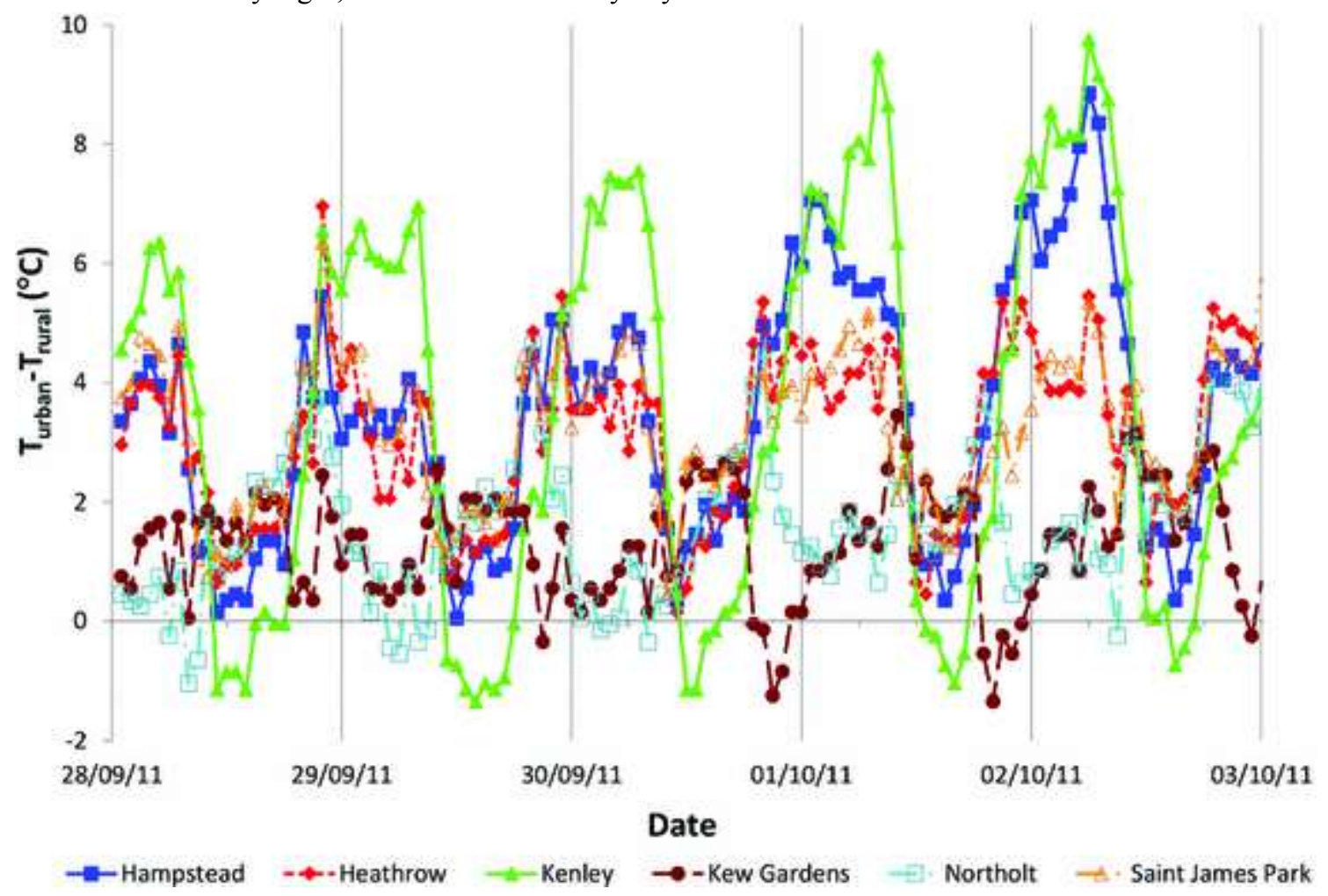

Fig. 4 Time series of the urban heat island intensity UHI for different locations in London, estimated as the temperature difference between several urban Met Office stations and the rural Chilbolton site, Turban-Trural

\subsection{Boundary layer evolution}

Given the low wind-speeds and clear sky conditions, the resulting big range in diurnal stability was expected to lead to clear differences between rural and UBL structure. This section considers the differences in evolution and structure of the boundary layer at both sites due to differing surface fluxes.

\subsubsection{Surface sensible heat flux}

Figure $\underline{5}$ shows incoming solar radiation for Chilbolton and WCC ( $\mathrm{SinCh}, \mathrm{SinWCC})$ and sensible heat flux measured at Chilbolton, WCC and BT (HCh,HwCC and HBT). It can be seen that conditions remained cloudless, highly unusual for the UK, which led to a strongly thermally driven flow. Given the differing heights $(5,18$ and 191 m respectively) 
and thus source areas of the flux instrumentation, emphasis will be given here to comparing the variability rather than magnitude of values. Nevertheless, HBT is higher than HwCC as it is located above the roughness sub-layer and has a much larger source area (i.e. approximately $2-22 \mathrm{~km}$ upwind of the Tower, depending on stability [15]), suggesting that the actual surface sensible heat flux in London is larger than for the rural surface, as expected. A strong diurnal cycle is seen at all sites, but there is a characteristic time lag between $\mathrm{HCh}$ and $\mathrm{HWCC}$ at the end of the day. Whilst $\mathrm{H}$ becomes positive at 08:00 for both sites, it becomes negative at around 17:00 at Chilbolton, and 20:00 for London. There is a slight decrease in peak fluxes across the first 4 days of the period, perhaps in response to the slightly decreased incoming solar radiation.

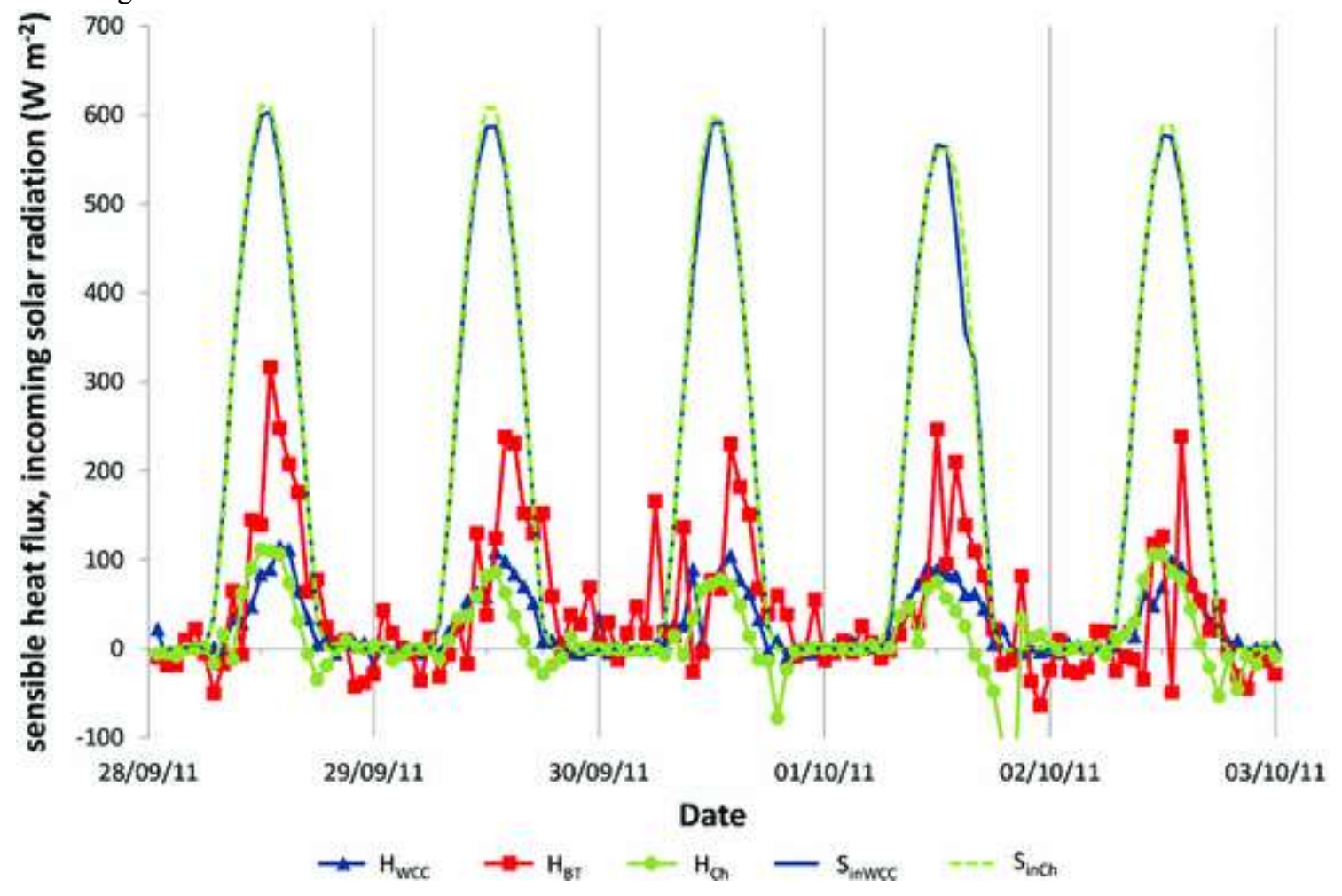

Fig. 5 Time series of sensible heat flux, $H$, at WCC, BT and Chilbolton sites and incoming solar radiation, $S_{i n}$, measured at WCC and Chilbolton

\subsubsection{Stability of the urban surface layer}

Figure $\underline{6}$ shows the hourly averaged potential temperature observed at the central London sites (WCC and BT) at $\mathrm{z}=18$ and $191 \mathrm{~m}$ respectively. Overall, the diurnal variation in temperature is similar each day. The minimum hourly averaged temperature for WCC increases at 06:00 each day, and for BT increases usually one hour later. Given that sunrise is at around 06:00 and shortwave incoming radiation increased measurably from 06:15 each day, this shows a lag in upward mixing of heat as the sun warms the surface. The Herstmonceux potential temperature interpolated to $191 \mathrm{~m}$ is added for comparison: it can be seen that the BT temperature tracks the Herstmonceux temperature well, indicating that warming between 28 and 29 September is widespread, and not just over the urban area. 


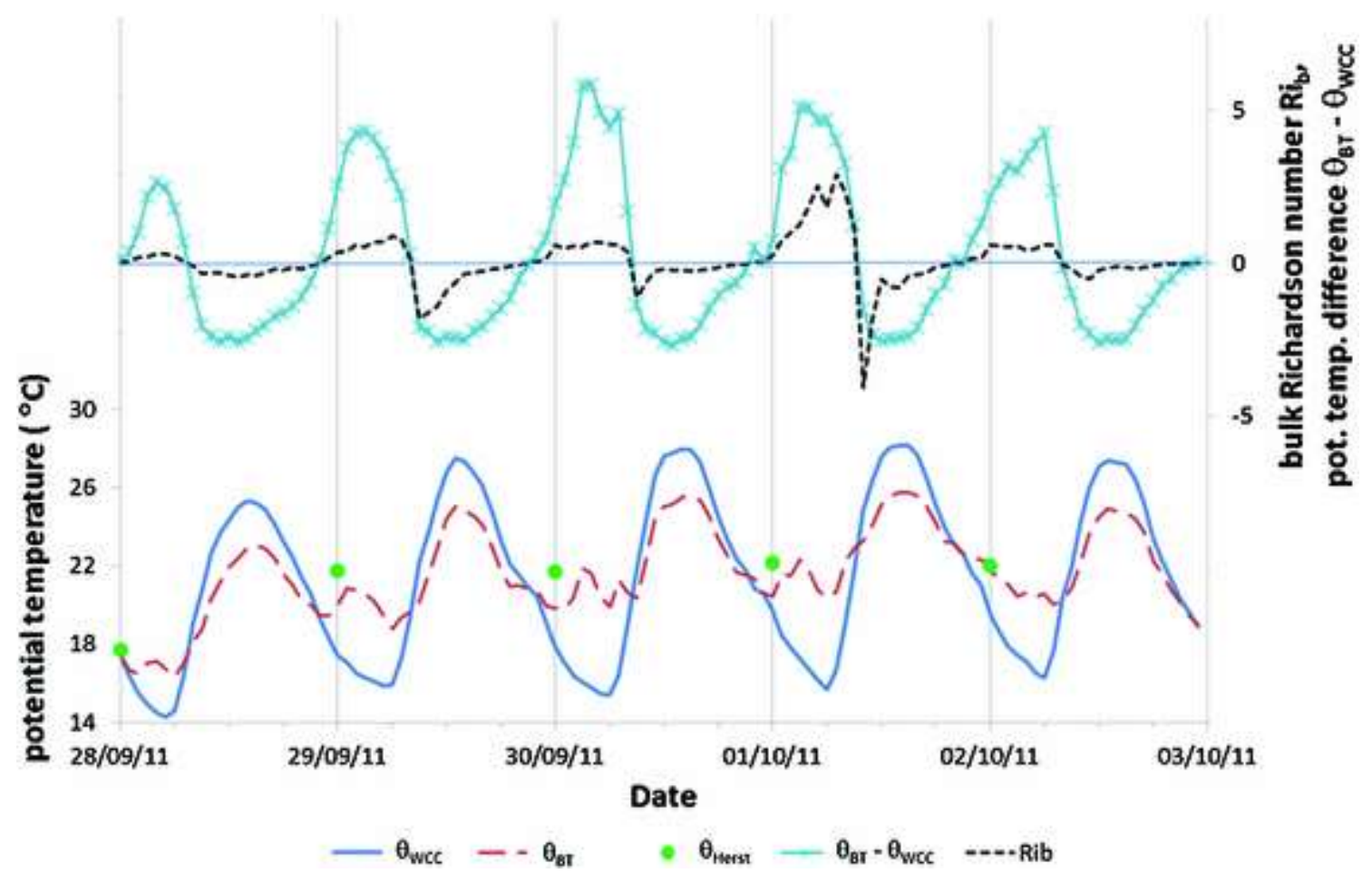

Fig. 6 Time series of potential temperature $\theta$ measured at central London sites WCC and BT, and from Herstmonceux radiosonde at 00:00. Also shown is potential temperature difference $\theta B T-\theta W C C$, and bulk Richardson number $R i b$

To determine the stability of the urban surface layer, Fig. $\underline{6}$ also shows the potential temperature difference $\theta \mathrm{BT}-\theta \mathrm{wCC}$, which indicates local static stability, and the bulk Richardson number, $R i b=(g \Delta \theta \Delta z) /(\theta-(\Delta u 2+\Delta v 2))$ which indicates dynamic stability. $\theta B T-\theta W C C$ reached a daytime unstable lapse rate of approximately $-2.5 \circ$ each day, but at night varied between 2.6。 and 5.8。, showing variability in the strength of the nocturnal inversion. The inversion was established at the same time each night, 22:00, indicating that it took approximately $2 \mathrm{~h}$ after the WCC heat flux initially became negative for an inversion of depth $200 \mathrm{~m}$ to be established. The dynamic stability parameter $R i b$ shows that on most days there are two stages - large, negative values as the boundary layer is first growing, then more constant values when the $\mathrm{CBL}$ is fully developed. Overnight, Rib increases slightly, showing large values even when $\theta B T-\theta W C C$ starts to reduce. This suggests that wind shear is important in determining night-time UBL stability.

\subsubsection{Mixing heights}

Figure 7 shows the mixing heights for London and Chilbolton (MHLon,MHCh) derived from Doppler lidar data. There is a clear difference between sites in magnitude and trend in $\mathrm{MH}$ over the period. Whilst maximum MHCh decreases, MHLon increases. These opposing trends are not explained by the trend in $\mathrm{H}$, which is a weak decrease. Given that the maximum urban temperatures by day are $27 \circ \mathrm{C}$ (Fig. $\underline{6}$ ), by applying a simple parcel ascent method to the regional potential temperature profiles shown in Fig. $\underline{3}$, the maximum boundary layer depth would be expected to increase from around 1,100 to $1,800 \mathrm{~m}$ (compared with the increase in mixing height from approximately 1,200 to $1,500 \mathrm{~m}$, Fig. 7). In comparison, the maximum rural temperatures were $25.5 \circ \mathrm{C}$, giving a maximum boundary layer depth which decreases throughout the period from approximately 1,300 to $800 \mathrm{~m}$. Hence, the "top-down" control of mixing height by the lapse rate has resulted in a factor of three difference in mixing height between rural and urban sites by the fifth day. 


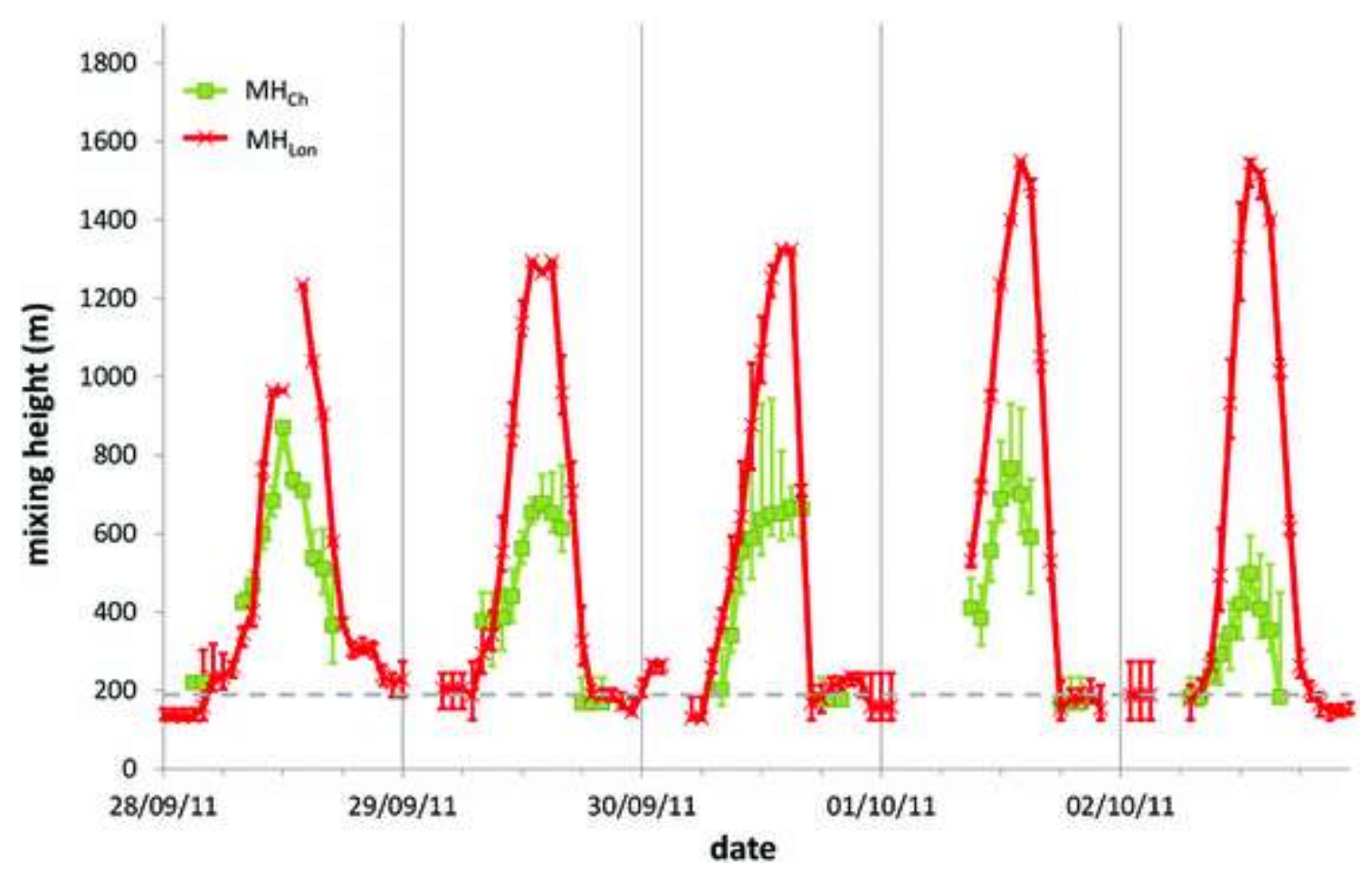

Fig. 7 Time series of mixing height $M H$ for London and Chilbolton

The diurnal cycle in $\mathrm{MH}$ for both sites is strongly linked to surface heat flux: morning growth is initiated at approximately the same time as the increase in $\mathrm{H}$ (08:00), but decay is delayed over the urban surface, with constant overnight $\mathrm{MH}$ at 20:00, when HwCC becomes negative. However, it should be recalled that the lowest observable mixing height for Chilbolton is $162 \mathrm{~m}$ while for London is $124 \mathrm{~m}$, therefore the night-time mixing heights are not well observed. Note that mixing height MHLon drops below the BT Tower during all nights - this may explain the higher variability in $\mathrm{HBT}$ and $\theta \mathrm{BT}$ as the sensors are at times near the top of a surface-based turbulent layer. MHLon was lower than the height of the BT Tower on $40 \%$ of hours (of which $15 \%$ were undetectable, lying below the $124 \mathrm{~m}$ gate).

\subsubsection{Diurnal cycle of the UBL}

In summary, the diurnal cycle in mixing height in this case is clearly linked to the diurnal cycle in sensible heat flux, with an extended decay period over the urban surface. MH magnitude depends on both the regional temperature profiles and surface temperature. The combination of the two factors has resulted in radically different trends in maximum mixing height for the two sites.

Despite the differences between the days, the diurnal cycle is similar. Figure $\underline{8}$ shows an ensemble average of the diurnal cycle for variables at the urban site, in order to define different phases in the evolution of the UBL. It includes a

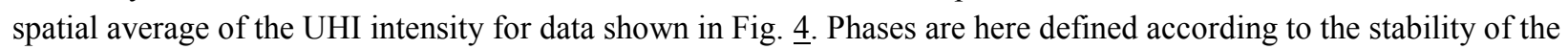
surface layer and development of the mixing height:

1 (09:00-12:00): rapid growth of convective UBL

2 (13:00-16:00): fully developed convective UBL

3 (17:00-20:00): decaying convective UBL

4 (21:00-24:00): transition from unstable to stable UBL

5 (01:00-08:00): stable UBL

Note that for the rural boundary layer, the decay of the convective boundary layer is rapid and in Phase 3 transition to stable boundary layer occurs. The question now arises as to how the resulting "Urban Convection Island" impacts on the structure of nocturnal wind profiles over the urban surface, which is explored in the next section. 


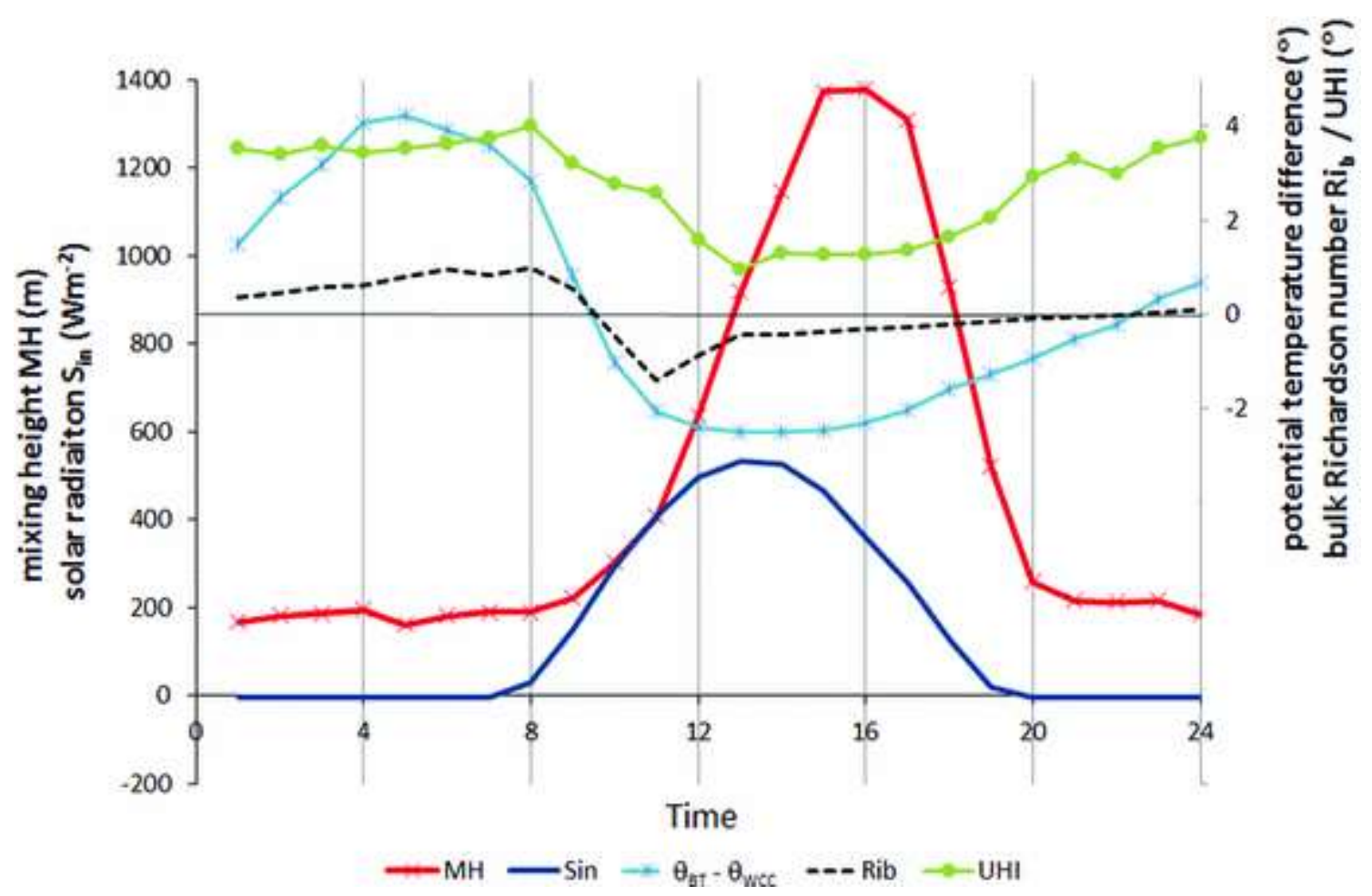

Fig. 8 Ensemble-averaged diurnal cycle of mixing height in London, $M H_{L o n}$, incoming solar radiation for WCC, $S_{i n}$, potential temperature difference, $\theta B T-\theta W C C$, bulk Richardson number, $R i b$ and $U H I$ intensity averaged over all urban stations

\section{Nocturnal wind profiles over the urban surface}

It was shown in Sect. 3.3.1 that stable conditions were observed at the rural site approximately $3 \mathrm{~h}$ before the urban site. A nocturnal jet can arise if conditions are stable enough, and decoupled flow aloft can execute inertial oscillations if stable conditions persist for long enough [23]. Assuming that the rural site stability observations are representative of the rural area surrounding London, the urban wind profiles are now analysed to test (a) whether a nocturnal jet is observed over the urban surface, despite persistent convective mixing, (b) whether its evolution is similar to a classical nocturnal jet. Unfortunately, only surface observations are available at the rural site for comparison as the lidar there does not have scanning capability.

\subsection{Evolution of nocturnal wind-speed and direction}

In Fig. 9 the time series of wind-speed for the UWCC and UCH sites and five selected lidar gates are shown (NB: UBT is well correlated with lidar observed windspeeds in the gate at $184 \mathrm{~m}$ and thus is not shown for clarity). Wind-speeds are generally low: UBT had a mean value of $5.1 \mathrm{~m} \mathrm{~s}^{-1}$ throughout the period, much lower the mean value of 7.7 m s-1 reported for the period May 2011-January 2012 [24]. The evening reduction in UwCC lags UCH by $1 \mathrm{~h}$, which is consistent with it being driven by convection persisting after sunset over the urban surface. For windspeeds detected aloft by the lidar, an opposing cycle can be seen: UwCC increases during the day as convection mixes down higher momentum air, and windspeed aloft in the mixed layer decreases and is near constant over the height range shown. There is evidence of a nocturnal jet in the urban wind profile: large wind shear is established overnight and maximum wind-speeds are observed at 244 or $304 \mathrm{~m}$. Measurements at $604 \mathrm{~m}$ are thus within the mixed layer by day 
and at times are above the jet by night. The data show that wind-speed aloft is highly variable between days despite similar forcing.

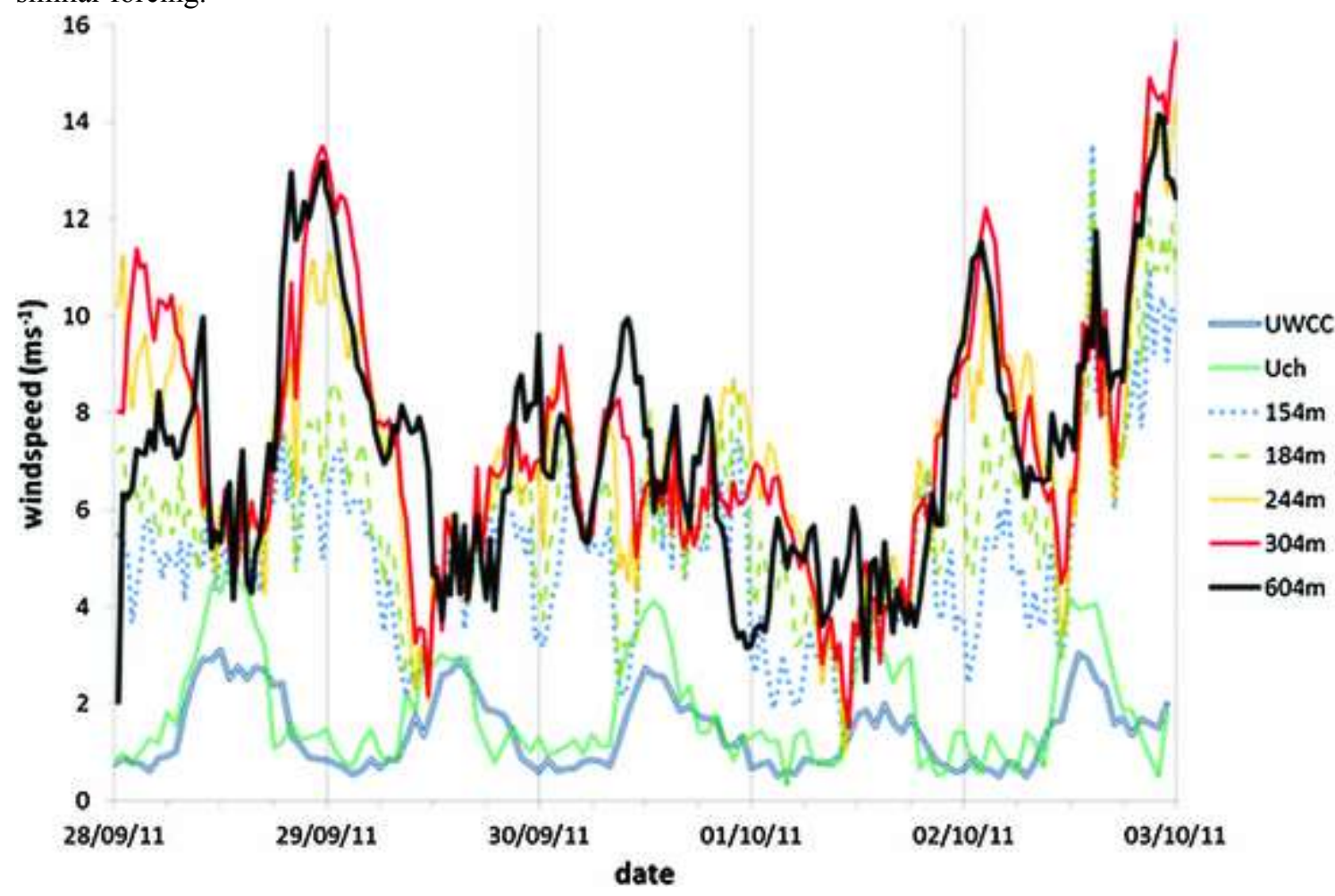

Fig. 9 Time series of wind-speed, $U$, at WCC, Chilbolton and for selected lidar gate heights

Given the opposing behaviour of the flow at the roof-top and flow aloft, Fig. 10 shows wind direction at WCC, $\varphi$ WCC, and at lidar gates $124 \mathrm{~m}, 184 \mathrm{~m}$ (BT Tower height) and $604 \mathrm{~m}$. The synoptically driven trend from south-easterly to south-westerly over the period can be seen, the exception being overnight values of $\varphi$ WCC, which are consistently northerly. This behaviour could be explained by drainage flow established through night-time cooling, given the gentle slope down towards the Thames river to the south, and more locally the slope of approximately 1० due to Primrose Hill, located $2 \mathrm{~km}$ to the north and $25 \mathrm{~m}$ higher than ground level at the WCC site. Flow at $124 \mathrm{~m}$ does not follow this trend, suggesting that a very shallow drainage current occurs. High resolution simulations with the UK Met Office Numerical Weather Prediction model for this case confirmed the presence of a drainage flow of depth approximately $100 \mathrm{~m}$, to be reported in a future publication (H. Lean, personal communication). Significant directional shear exists in the flow aloft at night, which evolves with time, suggesting that inertial oscillations are occurring, which is now tested. 


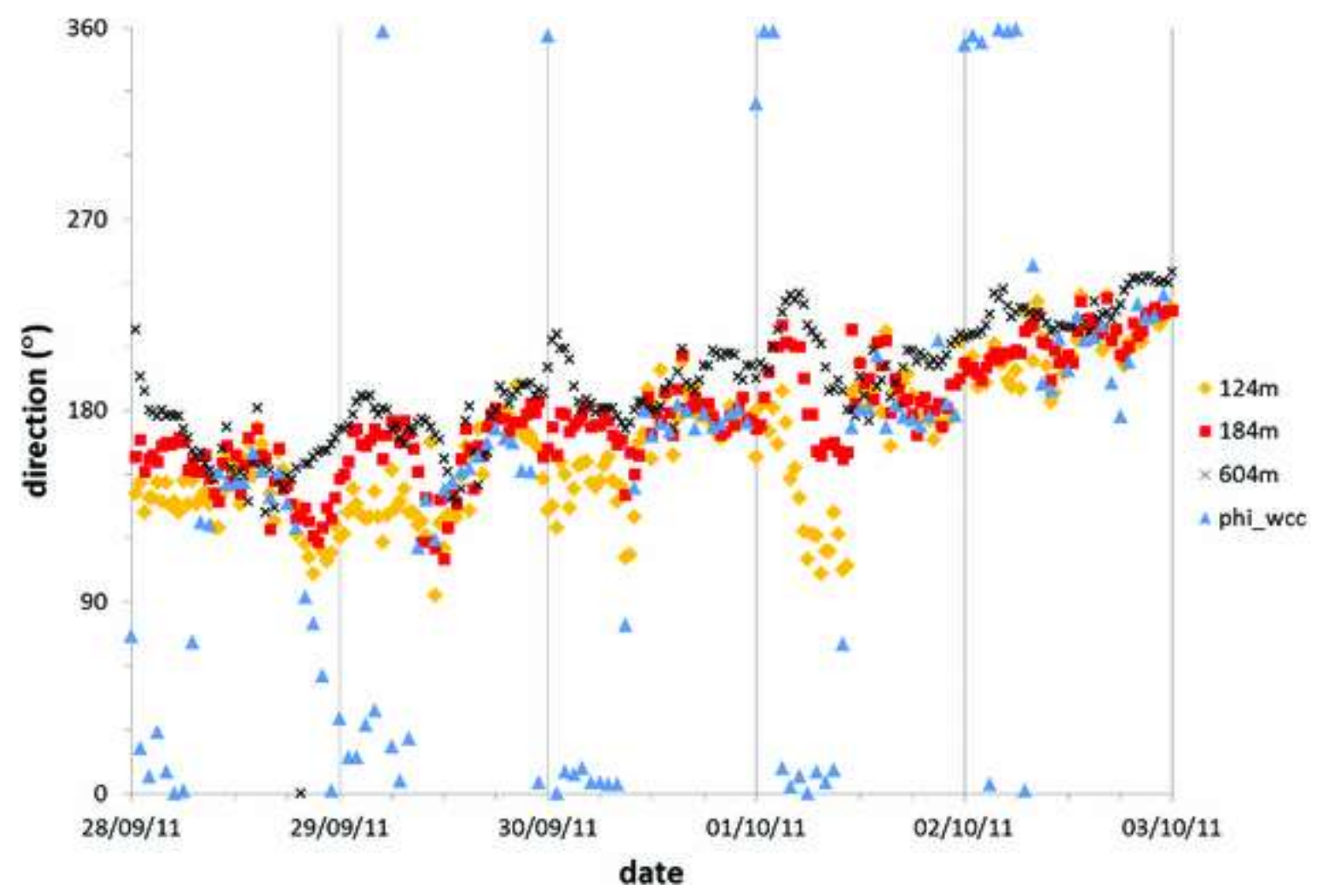

Fig. 10 Time series of wind direction, $\phi$, at WCC and for selected lidar gate heights

\subsection{Inertial oscillations}

Figure 11a- $\mathrm{d}$ shows hodographs for the overnight periods 28/29 September, 29/30 September, 30 September/01 October and 01/02 October, covering Phases 3, 4 and 5 from 17:00 to 08:00. The hodographs have been oriented such that increasing $\mathrm{u}$ component is a westerly and increasing $\mathrm{v}$ component is southerly, instead of the usual convention that $\mathrm{u}$ component aligns with the geostrophic vector. Note also that the starting vectors in the evening for each height are highlighted in black, and morning endpoint in white. It is clear in comparing all nights that flow at the WCC site follows the same evolution: shifting from southerly to northerly, and ending at a very similar point (magnitude $0.6 \mathrm{~m} \mathrm{~s}-1$ ), implying that the downslope flow within the urban canopy is very consistent and independent of forcing above. The downslope flow was established by around 20:00 each night, when $H W C C$ became negative. 
a

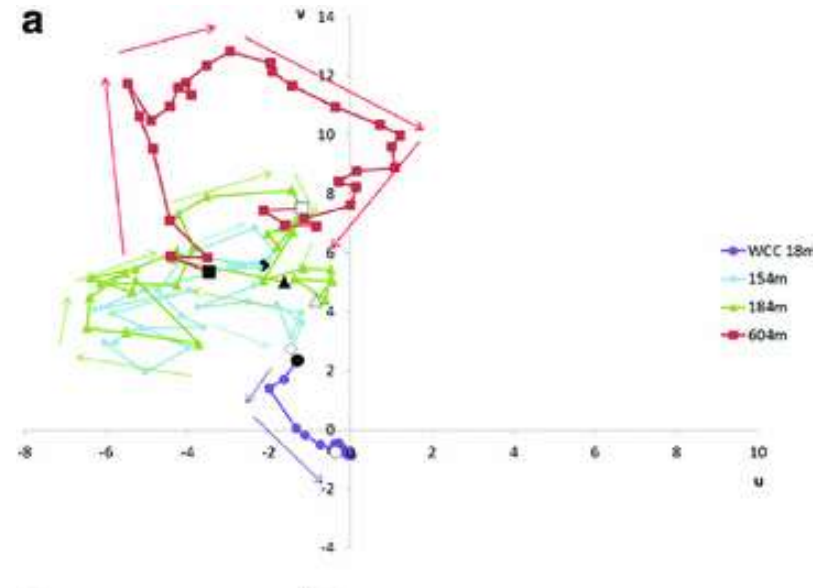

c

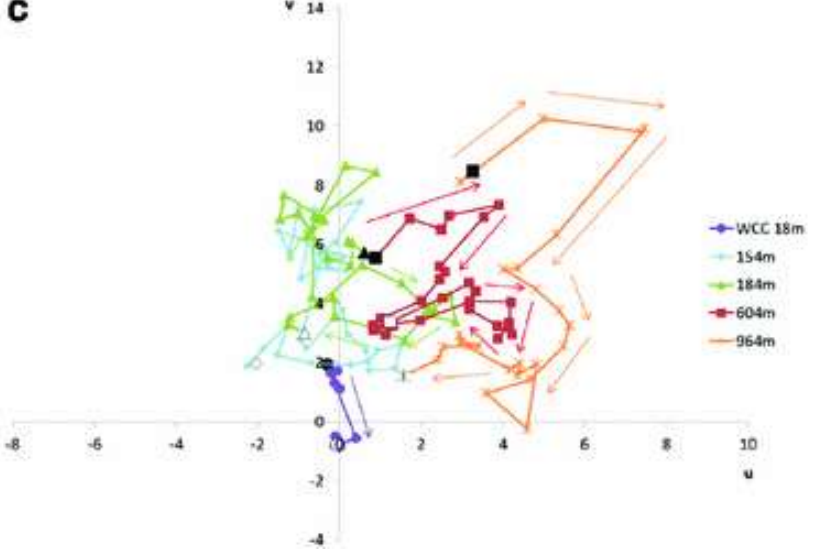

b $\quad$ (4)

จ 14

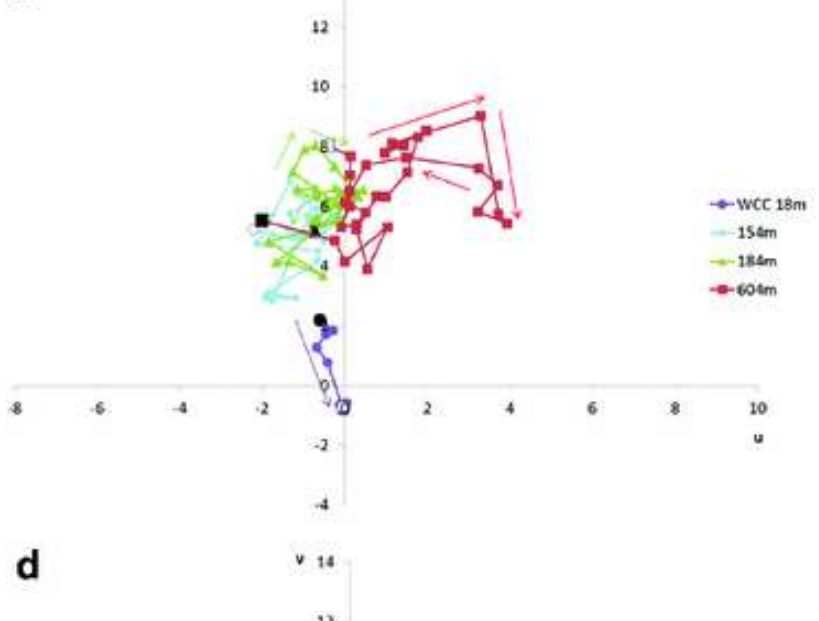

dis

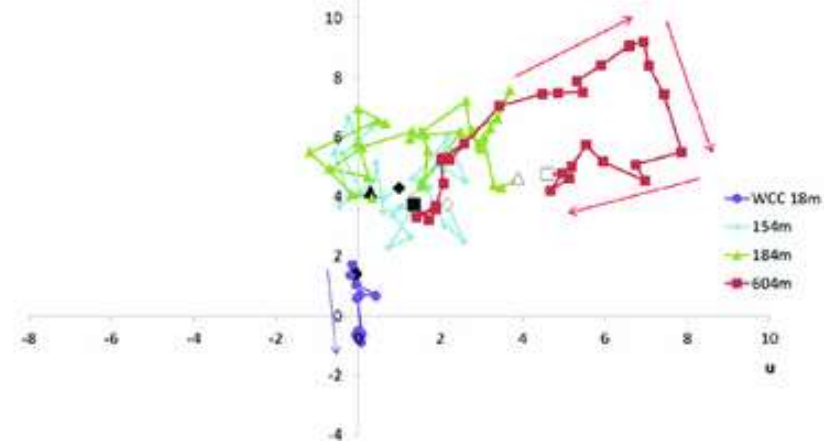

Fig. 11 Hodographs for overnight periods on a 28/29 September, b 29/30 September, c 30 September/01 October, $\mathbf{d} 01 / 02$ October. Axes are aligned such that increasing u component is a westerly and increasing $v$ component is southerly. Black symbols indicate starting vectors at 17:00 and white symbols indicate ending vectors at 08:00. Arrows indicate increasing time

Evidence of inertial oscillations (IO) is most obvious at $604 \mathrm{~m}$, which always lies above jet maximum (not shown, but between 184 and $334 \mathrm{~m}$ across all days). At $604 \mathrm{~m}$ a deflection was observed each night but it most resembles the classical IO on 28/29 September. On this night the wind vector at $184 \mathrm{~m}$ eventually also executed an IO after 00:00, suggesting that the lower layers were still coupled to the surface friction by turbulent mixing up to that time. The layer up to the BT Tower became stable from 23:00, when the mixing height was $238 \mathrm{~m}$, and a jet maximum first appeared at $394 \mathrm{~m}$. Figure 12 shows the wind profile throughout the evolution of the jet: during Phase 3 (decaying convection, 17:00-20:00), there was a significant acceleration of the flow above $200 \mathrm{~m}$. During Phase 4 (transition to stable BL, 21:00-24:00) the jet emerged; and during Phase 5 (stable UBL) the jet maximum gradually reduced in height to remain at $274 \mathrm{~m}$ from 04:00 to 07:30 (during which the mixing height was $203 \mathrm{~m}$ ), the reduction in height being classical IO behaviour. It is suggested here that the nocturnal jet formed over the rural surface and then was advected over the urban surface: its overall evolution suggests initiation at approximately 17:00 when the rural surface first became stable, but the delayed convection over the urban surface mixed down momentum, and the true jet structure was delayed until the urban surface layer became stable. 


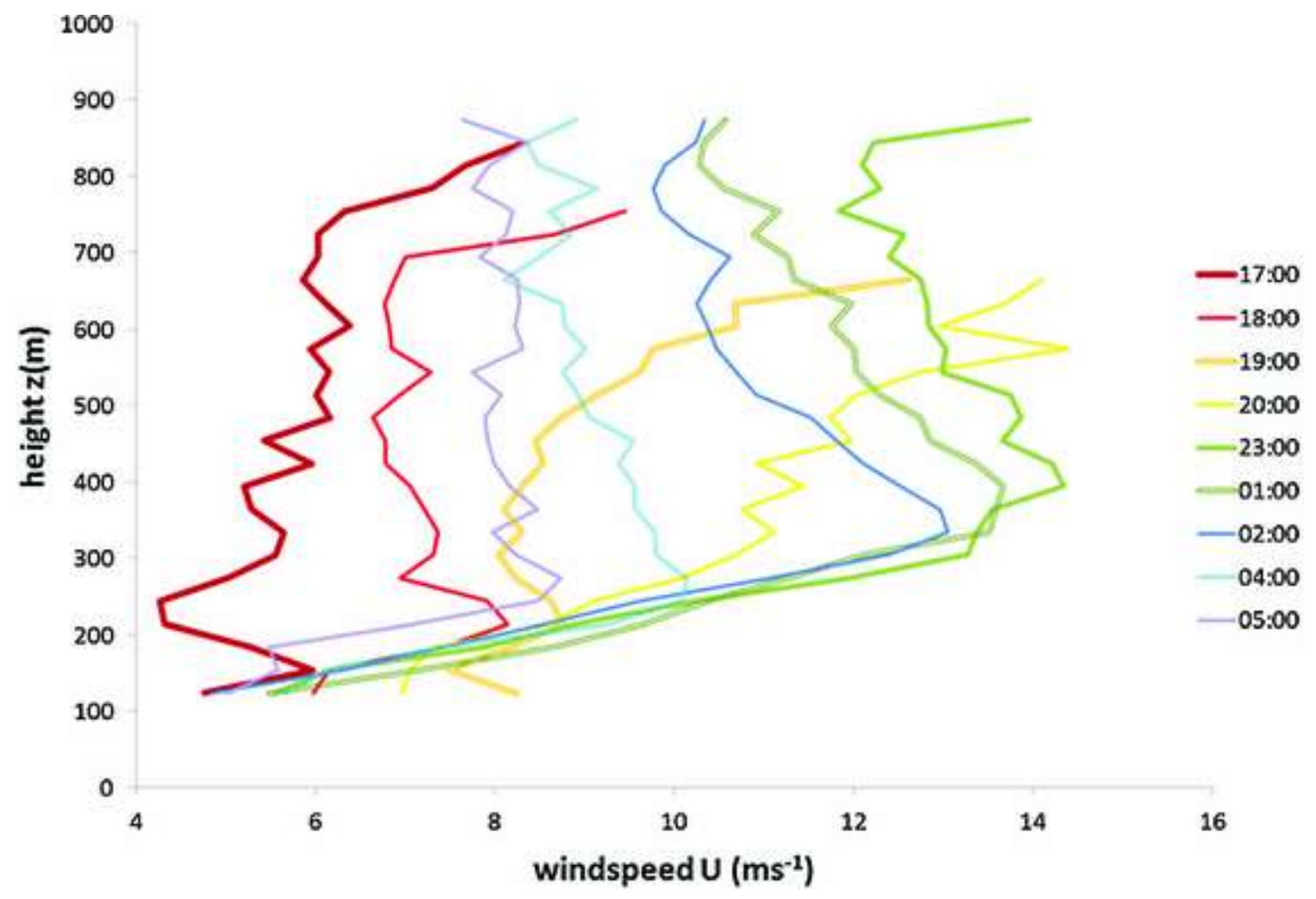

Fig. 12 Selected wind profiles showing jet evolution for overnight period 28/29 September

On other nights there was a somewhat disrupted IO, especially on 30/01, where lidar observations were available as high as $964 \mathrm{~m}$. Generally it is hard to observe clear IO motions in the "below jet layer", ie at 184 or $154 \mathrm{~m}$ - these levels are within or near the top of the mixing height but below jet maximum. Other studies at the rural Cabauw tower in the Netherlands $[\underline{25}, \underline{26}]$ have noted evidence of $\mathrm{IO}$ at these lower levels, but results presented here suggest that the urban surface heating and roughness can inhibit IO in the first part of the night in the lower layer, even if there is an IO above. In addition, for the Cabauw site, jets with clear inertial oscillations were not always observed, hence no conclusion can be drawn as to whether the "disrupted jet" behaviour shown here is peculiar to the urban surface. In summary, these observations suggest that nocturnal jets are initiated over the rural area; advection over the urban surface during the "urban convection island" causes downward mixing of momentum and thus a clear jet maximum may not be present; classical jet behaviour (i.e. inertial oscillation) continues over the urban area once stable conditions have been established.

\section{Wind and turbulence structure of the convective UBL}

Given that the nocturnal jet increases wind-speeds aloft during Phase 3 of the convective UBL, and persists throughout the night until Phase 1 of the next day, the impact of wind shear on turbulence structure during all phases of the convective UBL is now examined and compared with classical results.

\subsection{Wind profiles in the convective UBL}

Figure $13 \mathrm{a}-\mathrm{c}$ shows the average wind-speed profiles for Phases 1,2 and 3 each day for the urban site. Wind-speed profiles were scaled using the mixing height $\mathrm{MH}$ for each half hour period. To give height-averaged profiles for each Phase, the normalized height and wind-speed profiles were combined and ranked according to ascending $z / M H$ values and then ten-point bin averages were calculated. For Phase 1, (Fig. 13 a) whilst this averaging process has smoothed out any jet structure present, shear is present across the depth of the mixing height for all profiles, varying from strong (30 
September) to weak (28 September, 1 October). For Phase 2, as expected in a fully developed convective boundary layer, wind-speed profiles are generally uniform with height, except for 30 September and 2 October, when windspeeds are higher and there is considerable shear. In Phase 3, the decaying CBL, there is a sheared profile on 28 September, but on other days wind-speed is more uniform. Note that wind-speed magnitude increases slightly for most days in Phase 3, but drops radically for 2 October.
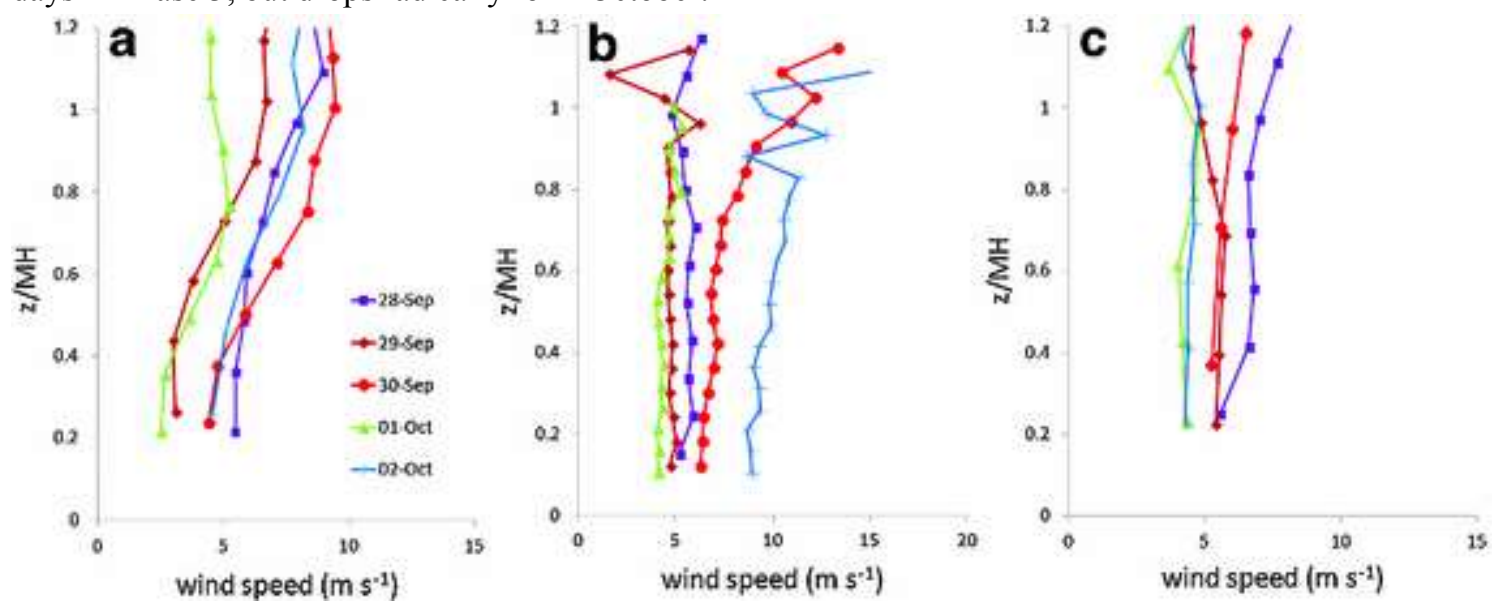

Fig. 13 Averaged wind-speed profiles for convective phases a 1, b 2 and $\mathbf{c} 3$ on each day for urban site. Height $z$ has been normalized using mixing height $M H$

\subsection{Variance, skewness and kurtosis profiles in the convective UBL}

Vertical velocity variance, $\sigma 2 w$, was calculated over hour-long periods to reduce the estimated systematic sampling error of the 90th percentile to less than $10 \%$, according to Lenschow et al. [27], and then averaged over three range gates in height to reduce random uncertainty [20]. Profiles were then scaled using the convective velocity scale $w_{*}=\left(g T 0 w^{\prime} T^{\prime} 0 z i\right) 1 / 3$

where $z i$ is the inversion height. The absolute surface temperature $T_{0}$ has been estimated by using the BT temperature; and surface heat flux was extrapolated from the BT Tower measured heat flux to the ground, using the relationship $[\underline{8}, \underline{14}]$. $w^{\prime} T^{\prime} z=w^{\prime} T^{\prime} 0(1-1.2 z z i)$

For the following analysis, the mixing height, $M H$, derived from the lidar was assumed to equal the inversion height $\mathrm{Zi}$. The normalized height $z / M H$ was thus calculated by dividing the actual height by the corresponding hourly mixing height, MH. To give height-averaged profiles for each Phase, the hourly variance profiles were combined and ranked according to ascending $z / M H$ values and then 10-point bin averages were calculated. Figure 14 compares the urban (Fig. 14a-c) and rural (Fig. 14d, e) profiles of $\sigma 2 w / w 2 *$ for periods when the heat flux was positive in phases 1,2 and 3 of all days. Note that heat flux was negative for the rural site in Phase 3 and therefore data are not included. Profiles are compared also with the semi-empirical profile of Lenschow et al. [28] and the non-entraining CBL profile of Sorbjan $[\underline{29}]$ to test how far from idealised CBL structure the UBL is in the different phases. 

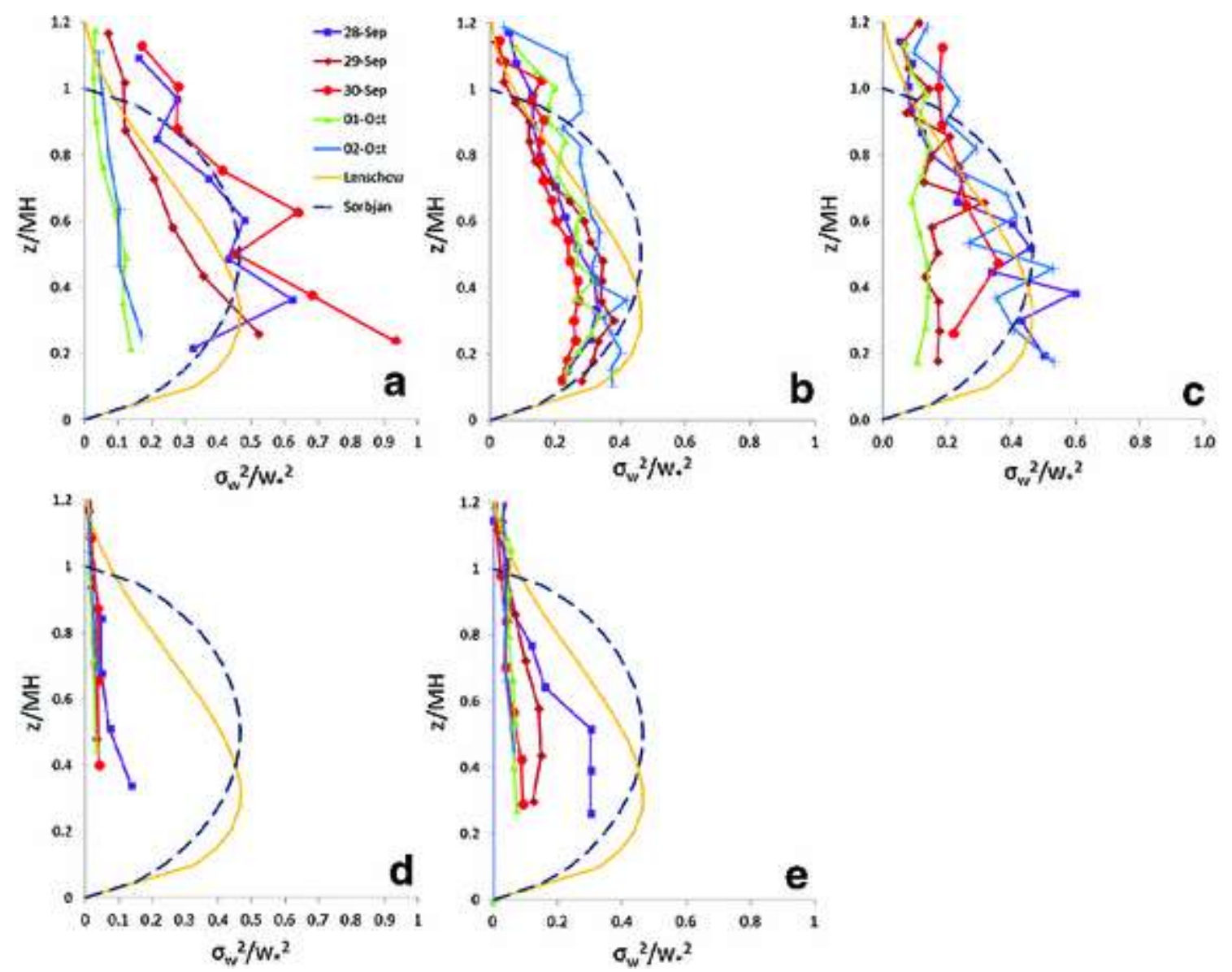

Fig. 14 Averaged, scaled, vertical velocity variance profiles, $\sigma 2 w / w 2 *$, for Phases 1,2 and 3 for the urban site (a-c) and for Phases 1 and 2 for the rural site (d, e) on each day. Height $z$ has been normalized using mixing height $M H$. Also shown are Lenschow et al. [28] and Sorbjan [29] semi-empirical profiles for the convective boundary layer

Skewness, $S k_{w}$, and kurtosis, $K_{w}$, profiles were also calculated, using $2 \mathrm{~h}$-long periods to reduce the estimated systematic sampling error of the 90 percentile to less than $15 \%$, according to [27]. As for $\sigma 2 w$, profiles were scaled using $z / M H$, ranked, then bin averaged to reduce scatter due to random error. Note that profiles shown in Figs. $\underline{15}$ and $\underline{16}$ are thus averages of only two 2 -h profiles within each 4 h-long Phase. Profiles are compared with the Most Convective (MC) and Least Convective (LC) cases of the Large Eddy Simulation Study by Lenschow et al. [30]. 

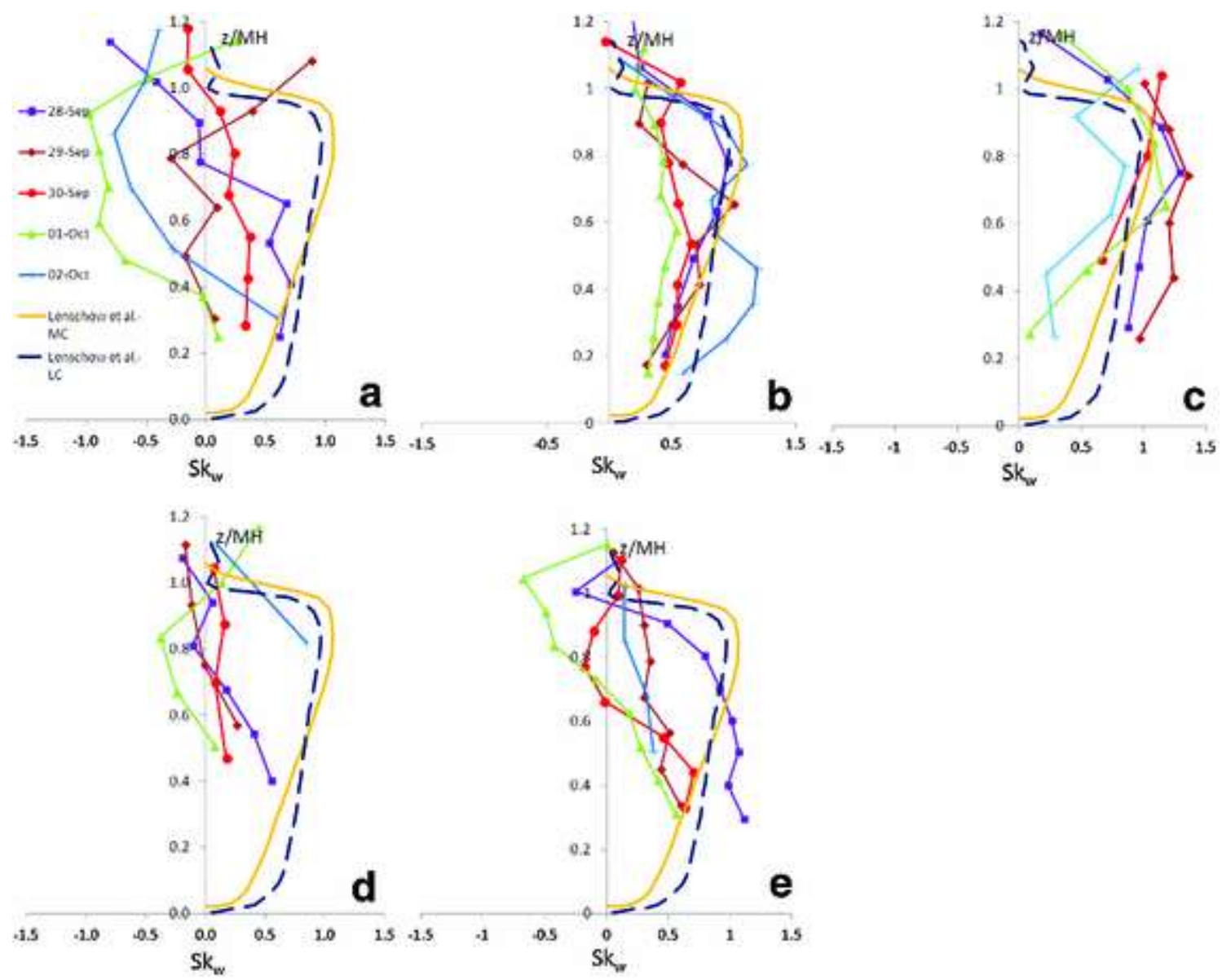

Fig. 15 Averaged vertical velocity skewness profiles, $S k w$, for Phases 1, 2 and 3 for the urban site (a-c) and for Phases 1 and 2 for the rural site (d, e) on each day. Height $z$ has been normalized using mixing height $M H$. Also shown are Most Convective (MC) and Least Convective (LC) profiles from Large Eddy Simulations, Lenschow et al. [푸] 

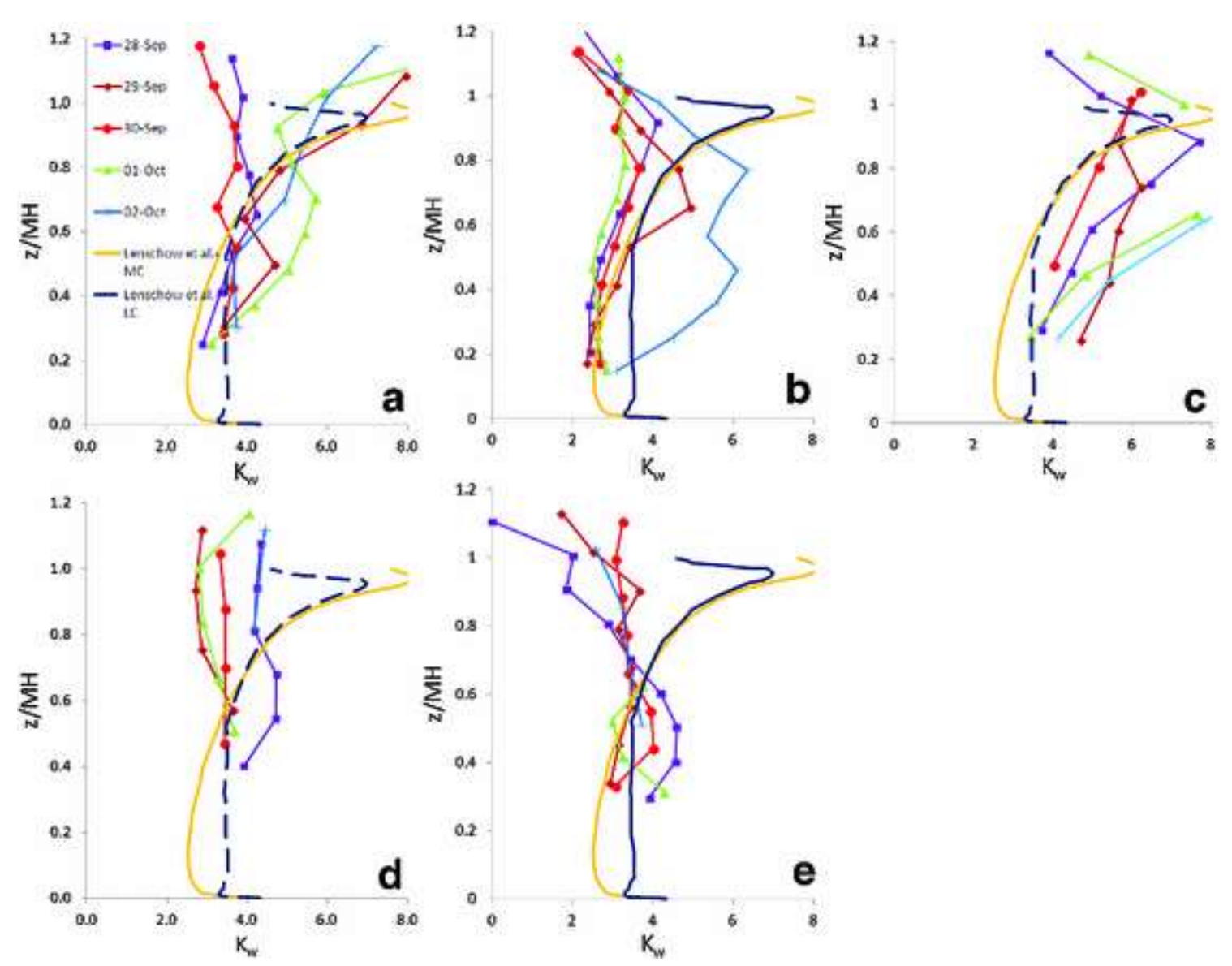

Fig. 16 Averaged vertical velocity kurtosis profiles, $K_{w}$, for Phases 1, 2 and 3 for the urban site (a-c) and for Phases 1 and 2 for the rural site (d, e) on each day. Height $z$ has been normalized using mixing height $M H$. Also shown are Most Convective (MC) and Least Convective (LC) profiles from Large Eddy Simulations, Lenschow et al. [무]

In the next section, each Phase is discussed in turn, and an integration of the features shown by the different turbulent moments is attempted.

\subsubsection{Phase 1: urban site}

For the urban site during Phase 1 it can be seen that days with large variance (Fig. 14) also have larger positive skewness values (Fig. 15, 28 and 30 September) and hence can be described as typically convective. In contrast, on 1 and 2 October the characteristic features of an "upside-down" boundary layer [ $\underline{31}]$ are apparent: in the presence of a jet, turbulence is generated aloft and transported downwards, leading to negative TKE fluxes and consequently negative skewness [32]. In addition, there is a monotonic decrease of variance with height, which is more characteristic of nearneutral, not convective, boundary layers. On all days a sheared windspeed profile was present (see Fig. 13a) but it is not apparent that the shear was stronger on 1 and 2 October compared to 28 and 30 September. The structure on 29 September lies between the two states.

\subsubsection{Phase 1: rural site}

For the rural site, variance profiles are uniformly small and monotonically decreasing with height, and skewness values are small and tend to be negative in the upper half of the boundary layer, but on days different to the UBL. These characteristics again suggest an "upside-down" boundary layer but in the absence of wind profile data, this cannot be 
corroborated. The most "convective" days are also 28 and 30 September, with the largest variance, positive skewness in the lower half of the boundary layer and larger kurtosis values (28 September).

In summary, the UBL exhibits most variability of state in Phase 1 (rapid CBL growth) and sensitivity to jet-produced wind shear.

\subsubsection{Phase 2: urban site}

In terms of structure, it is no surprise that the best collapse of variance data is shown for Phase 2 for the urban site. Profiles agree best with the Lenschow profile [ $\underline{28}]$ which is consistent with earlier analysis for fully developed CBLs over London done by [14] using the BT Tower turbulence data. The profile for 2 October has the least pronounced peak, but is the day with highest windspeeds ( $8-12 \mathrm{~m} \mathrm{~s}-1$, see Fig. $13 \mathrm{~b}$ ), suggesting that mixed convection is occurring. During Phase 2, skewness (Fig. 15b) and kurtosis (Fig. 16b) values agree with the Lenschow Most Convective (MC) case up to $z / M H \sim 0.5$.

\subsubsection{Phase 2: rural site}

Profiles in the fully developed phase for the rural site (Figs. $\underline{14}, \underline{15}, \underline{16}$ ) resemble the urban boundary layer in Phase 1: a mixture of "upside down" BL (particularly 30 September, 1 October), and convective ( 28 September). It can be seen that $\sigma 2 w / w 2 *$ is much smaller for the rural site than for London (Figs. $\underline{14}, \underline{15}, \underline{16}$ ), and is much smaller than the theoretically expected behaviour even in the fully developed CBL.

In summary, for the fully developed phase, the urban profiles resemble a "bottom-up", convective boundary layer more strongly than the rural profiles.

\subsubsection{Phase 3: urban site}

For Phase 3, there is more scatter in the variance profiles than for the fully developed phase (Fig. 14c cf.14b), but little evidence of "upside-down" behaviour: even for the smaller, monotonic variance profiles on 29 September and 2 October, skewness and kurtosis values are large and have similar tendencies to the Most Convective LES case. During this phase, there is little shear throughout the mixing height (Fig. 13 c) but the flow aloft can accelerate (e.g. $604 \mathrm{~m}, 28$ September, Fig. 12) as it starts to execute an inertial oscillation. In summary, this mixed boundary layer state is distinctly urban (convective layer below, jet above), as the rural boundary layer is stable during this phase.

\subsection{Impact of shear on kurtosis-skewness relationships}

The profiles of all three moments have together suggested that at times the increased shear due to the jet above has altered the turbulent structure of the convective layer below. Following Maurizi [33], kurtosis is now plotted against skewness and compared with limits predicted for free and forced convection according

to $K=\alpha(R i)\left(S_{2}+1\right)$ where $\alpha=3.3$ for shear-dominated and 2.4 for convection-dominated cases. This is done separately for the upper and lower parts of the boundary layer, as the comparison with LES results (Sect. $\underline{5.2}$ ) suggested different behaviours in each layer. Figure 17 shows each Phase for the UBL for lower (Fig. 17a-c) and upper (Fig. 17d-f) halves, and Fig. 18 shows Phase 1 and 2 for the rural boundary layer, lower (Fig. 18a, b) and upper (Fig. 18c, d). All points from each two-hour averaged profile are used in this analysis without bin averaging. 

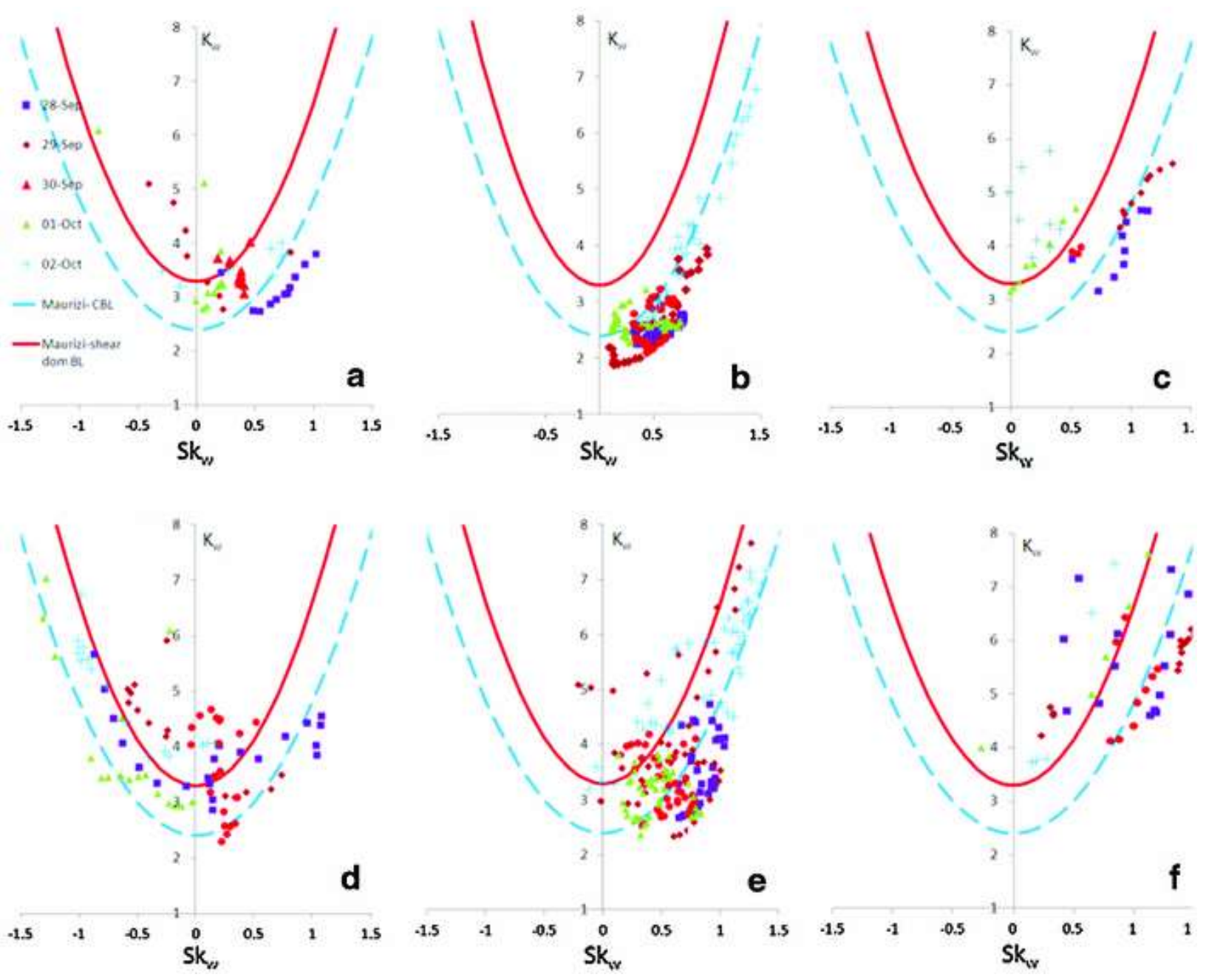

Fig. 17 Kurtosis-skewness relationships for the urban site for lower half of boundary layer, $z / M H<0.5$ Phase 1 (a), 2 (b) and 3 (c); and for upper half of boundary layer $z / M H>0.5$ Phase 1 (d), 2 (e) and 3 (f). Also shown are limiting relationships due to Maurizi [3] for the convection-dominated limit (blue dashed line) and shear-dominated limit (red solid line) 

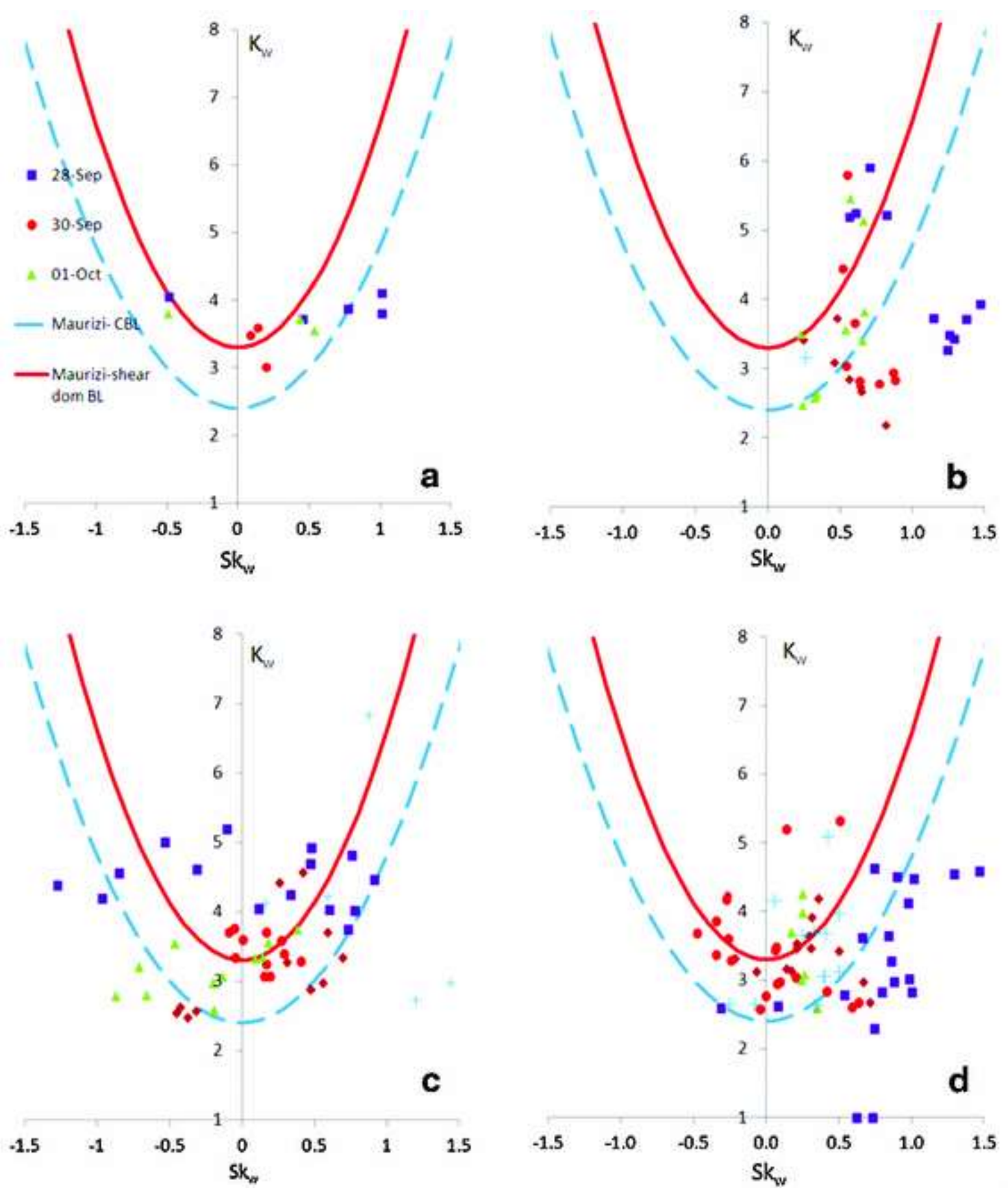

Fig. 18 Kurtosis-skewness relationships for the rural site for lower half of boundary layer, $z / M H<0.5$ Phase 1 (a) and 2 (b); and for upper half of boundary layer $z / M H>0.5$ Phase 1 (c) and 2 (d). Also shown are limiting relationships due to Maurizi [33] for the convection-dominated limit (blue dashed line) and shear-dominated limit (red solid line)

In general, during Phase 2 (fully developed), more data-points are closer to the convective limit than for the developing and decay stages. This is true for both urban and rural boundary layers (although there is more scatter for the rural site). There also tend to be more points closer to the shear limit in the upper half of the boundary layer for both sites. These results confirm the top-down influence of shear due to the jet in Phase 1 and 3, and suggest that there is still significant shear aloft during the fully developed boundary layer. This shear may be due to the strong subsidence inversion shown in Fig. $\underline{3}$, but cannot be confirmed as there are insufficient aerosol targets for the lidar to detect data above the boundary layer.

More specifically, data for the urban site on 28 September are particularly well ordered, following the convective limit in the lower half (i.e. Fig. 17a-c), and the shear limit in Phase 1 for the upper half (Fig. 17d). Data for the fully developed boundary layer on 2 October mostly follow the convective limit for kurtosis across the range of skewness 
(Fig. 17b, e), even though the kurtosis values seemed unusually large in Fig. 16 b. Given that this is the day with the highest wind-speeds, surface layer shear appears to play little role in influencing turbulence structure in the rest of the boundary layer. This is an interesting result: the warmer urban surface leads to strong buoyant production of turbulence which still dominates over shear production, despite increased surface roughness.

In summary, for the UBL, average day-time wind profiles are still strongly sheared due to the nocturnal jet during Phase 1 of rapid CBL growth. Second, third and fourth order turbulence moments are all affected and profiles reflect an "upside-down" boundary layer. The fully developed Phase 2 for the urban site resembles a "classical" convective boundary layer more than for the rural site. In the "Urban Convective Island" Phase 3, strong convection still occurs with some influence from shear aloft, depending on jet strength.

\section{Conclusions}

This paper has presented new observations of the structure of flow and turbulence above both an urban and rural surface and has investigated how much UBL structure deviates from classical behaviour during a strong UHI event. Overall, some key conclusions emerge:

(1) Boundary layer evolution for both rural and urban sites is determined by the diurnal cycle in sensible heat flux, with an extended decay period of $4 \mathrm{~h}$ for the urban convective boundary layer. This is referred to as the "Urban Convective Island" as the surrounding rural area is already stable at this time. Whilst it has previously been established that surface sensible heat flux remains positive after sunset in urban areas, this study has quantified the turbulence characteristics of the resulting convective layer, its depth dropping on average from approximately 1,400 to $200 \mathrm{~m}$ during this phase.

(2) Maximum magnitude of the mixing height depends not on sensible heat flux magnitude, but on the combination of regional temperature profiles and surface temperature. In this case study, due to multiple inversion layers in the ambient potential temperature profile, and a UHI intensity of $1.5 \circ \mathrm{C}$, the urban mixing height increases over the period whilst the rural mixing height decreases, resulting in a factor of three difference by the fifth day. This implies the need for accurate regional boundary conditions when modelling city scale urban climate processes.

(3) Analysis of night-time urban wind profiles suggests that nocturnal jets are initiated over the rural area when it becomes stable; they advect over the urban surface during the "Urban Convection Island", which causes downward mixing of momentum and thus a clear jet maximum is not present. Once stable conditions are established over the urban area, a jet maximum undergoing inertial oscillations can be observed aloft. However, at the level of the urban canopy, a decoupled layer can form during stable conditions due to downslope drainage currents. This emphasizes the importance of orography in the urban area, as it still plays an important role in determining flow under stable conditions, despite the extra roughness of the urban canopy.

(4) Due to the presence of the nocturnal jet, average day-time wind profiles can still be strongly sheared in the rapid, morning phase of convective boundary layer growth. Analysis of turbulent moments (variance, skewness and kurtosis) has shown corresponding "upside-down" boundary layer characteristics on some mornings. For the fully developed mid-day phase, the UBL better resembles classical convective boundary layer profiles than for the rural site, which may be more strongly impacted by the subsidence inversion (and corresponding wind shear aloft) associated with the high pressure system in this case. For the "Urban Convective Island" phase, turbulence structure still resembles a classical convective boundary layer but with some influence from shear aloft, depending on jet strength.

(5) Operating the Doppler lidar in a mixture of vertical stare and Doppler Beam Swinging (DBS) mode allowed for mean wind vector profiles and vertical velocity turbulence structure to be determined with reasonable accuracy for each averaging period. The combination of both scan modes allows detailed insight into the impact of stability on boundary layer evolution. 


\section{Acknowledgments}

Thanks to Rosy Wilson, Mike Stroud and John Lally for technical support during the ACTUAL project. Thanks to Chris Walden and Darcy Ladd for providing technical information about Chilbolton Observatory meteorological sensors. Thanks go to Steve Neville at Westminster City Council for permission to site instrumentation at the WCC rooftop site, and to BT for use of the BT Tower. The ACTUAL Project was funded under Engineering and Physical Sciences Research Council Grant Number EP/G022938/1 (www. actual.ac.uk).

\section{References}

1. Mestayer PG, Durand P, Augustin P et al (2005) The urban boundary-layer field campaign in Marseille (UBL/CLUESCOMPTE): set-up and first results. Bound Layer Meteorol 114:315-365

2. Masson V, Gomes L, Pigeon G et al (2008) The canopy and aerosol particles interactions in Toulouse urban layer (CAPITOUL) experiment. Meteorol Atmos Phys 102(3-4):135-157

3. Pal S, Xuref-Remy I, Ammoura L et al (2012) Spatio-temporal variability of the atmospheric boundary layer depth over the Paris agglomeration: an assessment of the impact of the urban heat island intensity. Atmos Environ 63:261275

4. Kallistratova MA, Kouznetsov RD (2011) Low-level jets in the Moscow region in summer and winter observed with a sodar network. Bound Layer Meteorol 143(1):159-1755. Wang Y, Klipp C, Garvey DM, Ligon DA, Williamson CC, Chang SS, Newsom RK, Calhoun R (2007) Nocturnal low-level-jet-dominated boundary layer observed by a Doppler lidar over Oklahoma City during JU2003. J Appl Meteorol Climatol 46(12):2098-2109

6. Lundquist JK, Mirocha JD (2008) Interaction of nocturnal low-level jets with urban geometries as seen in Joint Urban 2003 data. J Appl Meteorol Climatol 47(1):44-58

7. Roth M (2000) Review of atmospheric turbulence over cities. Q J R Meteorol Soc 126:941-990

8. Barlow JF, Dunbar TM, Nemitz EG, Wood CR, Gallagher MW, Davies F, O’Connor E, Harrison RM (2011)

Boundary layer dynamics over London, UK, as observed using Doppler lidar during REPARTEE-II. Atmos Chem Phys 11:2111-2125

9. Seibert P, Geyrich F, Gryning SE, Joffre S, Rasmussen A, Tercier P (2000) Review and intercomparison of operational methods for the determination of the mixing height. Atmos Environ 34:1001-1027

10. Emeis S, Schäfer K, Münkel C (2008) Surface-based remote sensing of the mixing-layer height: a review. Meteorol Z 17(5):621-630

11. Otkin JA, Hartung DC, Turner DD et al (2011) Assimilation of surface-based boundary layer profiler observations during a cool-season weather event using an observing system simulation experiment. Part I: analysis impact. Mon Weather Rev 139(8):2309-2326

12. Collier CG, Davies F, Bozier KE, Holt AR, Middleton DR, Pearson GN, Siemen S, Willetts DV, Upton GJG, Young RI (2005) Dual-Doppler lidar measurements for improving dispersion models. B Am Meteorol Soc 86:825-838 13. Calhoun R, Heap R, Princevac M, Newsom R, Fernando H, Ligon D (2006) Virtual towers using coherent Doppler lidar during the Joint Urban 2003 dispersion experiment. J Appl Meteorol Climatol 45:1116-1126

14. Wood CR, Lacser A, Barlow JF, Padhra A, Belcher SE, Nemitz E, Helfter C, Famulari D, Grimmond CSB (2010) Turbulent flow at $190 \mathrm{~m}$ above London during 2006-2008: a climatology and the applicability of similarity theory. Bound Layer Meteorol 137:77-96

15. Helfter C, Famulari D, Phillips GJ, Barlow JF, Wood CR, Grimmond CSB, Nemitz E (2011) Controls of carbon dioxide concentrations and fluxes above central London. Amos Chem Phys 11:1913-1928

16. Lane SE, Barlow JF, Wood CR (2013) An assessment of a three-beam Doppler lidar wind profiling method for use in urban areas. J Wind Eng Ind Aerodyn 119:53-59

17. Barlow JF, Harrison J, Robins AG, Wood CR (2011) A wind-tunnel study of flow distortion at a meteorological sensor on top of the BT Tower, London. UK. J Wind Eng Ind Aerodyn 99(9):899-907

18. Barlow JF, Dobre A, Smalley RJ, Arnold SJ, Tomlin AS, Belcher SE (2009) Referencing of street-level flows: results from the DAPPLE 2004 campaign in London, UK. Atmos Environ 43:5536-5544

19. Wilczak JM, Oncley SP, Stage SA (2001) Sonic anemometer tilt correction algorithms. Bound Layer Meteorol 99:127-150 
20. Hogan RJ, Grant ALM, Illingworth AJ, Pearson GN (2009) Vertical velocity variance and skewness in clear and cloud-topped boundary layers as revealed by Doppler lidar. Q J R Meteorol Soc 135:635-643

21. Oke TR (1987) Boundary layer climates, 2nd edn. Routledge, London

22. Bohnenstengel SI, Evans S, Clark PA, Belcher SE (2011) Simulations of the London urban heat island. Q J R Meteorol Soc 137(659):1625-1640

23. Blackadar AK (1957) Boundary layer wind maxima and their significance for the growth of nocturnal inversions.

Bull Am Meteorol Soc 38:283-290

24. Drew DR, Barlow JF, Lane SE (2013) Observations of wind speed profiles over Greater London, UK, using a Doppler lidar. J Wind Eng Ind Aerodyn 121:98-105

25. Van der Wiel B, Moene G, Steeneveld G, Baas P, Bosveld FC, Holtslag AAM (2010) A conceptual view on inertial oscillations and nocturnal low-level jets. J Atmos Sci 67(8):2679-2689

26. Baas P, van den Wiel B, van den Brink, Holtslag AAM (2012) Composite hodographs and inertial oscillations in the nocturnal boundary layer. Q J R Meteorol Soc 138(663):528-535

27. Lenschow DH, Mann J, Kristensen L (1994) How long is long enough when measuring fluxes and other turbulence statistics. J Atmos Ocean Technol 11:661-673

28. Lenschow DH, Wyngaard JC, Pennell WT (1980) Mean-field and second-moment budgets in a baroclinic, convective boundary layer. J Atmos Sci 37:1313-1326

29. Sorbjan Z (1990) Similarity scales and universal profiles of statistical moments in the convective boundary layer. J Appl Meteorol 30:1565-1583

30. Lenschow DH, Lothon M, Mayor SD, Sullivan P, Canut G (2012) A comparison of higher-order vertical velocity moments in the convective boundary layer from lidar with in-situ measurements and large-eddy simulation. Bound Layer Meteorol 143(1):107-123

31. Mahrt L (1999) Stratified atmospheric boundary layers. Bound Layer Meteorol 90(3):375-396

32. Banta RM, Pichugina YL, Brewer WA (2006) Turbulent velocity-variance profiles in the stable boundary layer generated by a nocturnal low-level jet. J Atmos Sci 63(11):2700-2719

33. Maurizi A (2006) On the dependence of third- and fourth-order moments on stability in the turbulent boundary layer. Nonlinear Process Geophys 13:119-123 